JOURNAL OF INTEGRAL EQUATIONS

AND APPLICATIONS

Volume 14, Number 3, Fall 2002

\title{
AN ANALYTIC SEMIGROUP SETTING FOR A CLASS OF VOLTERRA EQUATIONS
}

\author{
PH. CLÉMENT, W. DESCH AND K.W. HOMAN
}

ABSTRACT. We provide a semigroup setting for the scalar Volterra integrodifferential equation of convolution type

$$
\begin{aligned}
\frac{d}{d t} \int_{-\infty}^{t} a(t-s) u(s) d s & =f(t), \quad t>0, \\
u(t) & =u_{0}(t), \quad t \leq 0,
\end{aligned}
$$

with a completely monotonic kernel $a:(0, \infty) \rightarrow \mathbf{R}$. Using the analyticity of the semigroup and interpolation methods we analyze regularity of solutions.

1. Introduction. The aim of this paper is to provide a semigroup setting for the scalar Volterra integrodifferential equation of convolution type

$$
\begin{aligned}
\frac{d}{d t} \int_{-\infty}^{t} a(t-s) u(s) d s & =f(t), \quad t>0 \\
u(t) & =u_{0}(t), \quad t \leq 0
\end{aligned}
$$

where the kernel $a:(0, \infty) \rightarrow \mathbf{R}$ is assumed to be completely monotonic and such that $\int_{0}^{1} a(t) d t<\infty$ and $a(0+)=+\infty$, and where the functions $u_{0}:(-\infty, 0] \rightarrow \mathbf{R}$ and $f:[0, \infty) \rightarrow \mathbf{R}$ are given.

There is a classical way to treat problem (1): Let $b:(0, \infty) \rightarrow \mathbf{R}$ be the function resolvent of the first kind of $a$, which means that $b$ satisfies

$$
\int_{0}^{t} a(t-s) b(s) d s=1, \quad t>0,
$$

see [9, p. 158]. The function $b$ is completely monotonic and satisfies $\int_{0}^{1} b(t) d t<\infty$ and $b(0+)=+\infty$. If $u_{0}$ is identically zero and if

Received by the editors on December 10, 2001, and in revised form on September 25, 2002.

Copyright (C)2002 Rocky Mountain Mathematics Consortium 
$f$ is locally integrable, then problem (1) admits a unique solution $u: \mathbf{R} \rightarrow \mathbf{R}$ and

$$
u(t)= \begin{cases}\int_{0}^{t} b(t-s) f(s) d s & t>0, \\ 0, & t \leq 0 .\end{cases}
$$

A typical example of the kernel is

$$
a(t)=\frac{t^{-\alpha}}{\Gamma(1-\alpha)}, \quad t>0,
$$

with $0<\alpha<1$, in which case the lefthand side of (1) corresponds to the fractional derivative of order $\alpha$ of $u$ and

$$
b(t)=\frac{t^{\alpha-1}}{\Gamma(\alpha)}, \quad t>0
$$

To our knowledge there is no general theory concerning problem (1) with given initial data $u_{0}$ defined on $(-\infty, 0]$.

In this paper we treat existence and regularity of solutions to (1) within the framework of analytic semigroups and interpolation spaces. This sheds a new light on results obtained by more direct methods. In particular it opens access to a powerful analytic machinery:

There is a well-established theory of stochastic perturbation of analytic semigroups, as for example in [5], which will be useful when the forcing term $f$ in (1) is replaced by a stochastic process.

Some concepts, like Kolmogorov equations, depend on a state space formulation of the underlying process.

We expect that our method can be extended from the scalar case to more ambitious Hilbert space valued problems.

Frequently, the price for embedding integral or delay equations in a semigroup framework is some loss of regularity. Our method, as the example of fractional derivatives shows, yields regularity results which are close to optimal.

Our approach consists of rewriting (1) as an ordinary differential equation in an appropriate infinite dimensional Hilbert space, using the complete monotonicity of the kernel and Bernstein's theorem. The 
semigroup approach based on the complete monotonicity of the kernel has been initiated in [8], has been used in the context of viscoelasticity in [6], and has been generalized to a larger class of kernels in [14]. In contrast to other semigroup approaches for Volterra equations, as for example in $[\mathbf{1 1}, \mathbf{9}]$, this one leads to a quasi-contractive, analytic semigroup.

Heuristically the approach can be explained as follows. Let $\nu$ be the unique nonnegative Borel measure on $[0, \infty)$ such that

$$
a(t)=\int_{[0, \infty)} e^{-\kappa t} \nu(d \kappa), \quad t>0,
$$

see Bernstein's theorem $[\mathbf{1 7}$, p. 161]. We define the functions $\psi$ : $[0, \infty) \times[0, \infty) \rightarrow \mathbf{R}$ and $\psi_{0}:[0, \infty) \rightarrow \mathbf{R}$ by respectively

$$
\begin{aligned}
\psi(t, \kappa) & :=\int_{-\infty}^{t} e^{-\kappa(t-s)} u(s) d s, \quad t \geq 0, \kappa \geq 0, \\
\psi_{0}(\kappa) & :=\int_{0}^{\infty} e^{-\kappa s} u_{0}(-s) d s, \quad \kappa \geq 0,
\end{aligned}
$$

where $u$ is the solution to (1). Note that $\psi$ satisfies

$$
\begin{aligned}
\frac{\partial}{\partial t} \psi(t, \kappa) & =u(t)-\kappa \psi(t, \kappa), \quad t>0, \kappa \geq 0, \\
\psi(0, \kappa) & =\psi_{0}(\kappa), \quad \kappa \geq 0 .
\end{aligned}
$$

Then we have for $t>0$

$$
\begin{aligned}
f(t) & =\frac{d}{d t} \int_{-\infty}^{t} a(t-s) u(s) d s \\
& =\frac{d}{d t} \int_{-\infty}^{t}\left(\int_{[0, \infty)} e^{-\kappa(t-s)} \nu(d \kappa)\right) u(s) d s \\
& =\frac{d}{d t} \int_{[0, \infty)}\left(\int_{-\infty}^{t} e^{-\kappa(t-s)} u(s) d s\right) \nu(d \kappa) \\
& =\frac{d}{d t} \int_{[0, \infty)} \psi(t, \kappa) \nu(d \kappa) \\
& =\int_{[0, \infty)} \frac{\partial}{\partial t} \psi(t, \kappa) \nu(d \kappa) \\
& =\int_{[0, \infty)}(u(t)-\kappa \psi(t, \kappa)) \nu(d \kappa) .
\end{aligned}
$$


Hence, $\psi$ satisfies (4) with side-constraint

$$
\int_{[0, \infty)}(u(t)-\kappa \psi(t, \kappa)) \nu(d \kappa)=f(t), \quad t>0 .
$$

A novelty with respect to $[\mathbf{8}]$ and $[\mathbf{6}]$ is that in our situation the solution $u$ to problem (1) does not belong to the state space of $\psi$, but it can be computed from the state space by means of an unbounded linear functional.

The paper is organized as follows: Section 2 contains some of the notations used throughout this paper. Section 3 states the main results. In Section 4 we set up a state space $H$ and a linear operator $A: D(A) \subseteq H \rightarrow H$ and prove that $A$ generates an analytic semigroup on $H$. Section 5 treats the homogeneous case of (1) when $u_{0}$ is so good that the solution to the abstract problem (4)-(5) remains in $D(A)$. Section 6 provides necessary tools from interpolation spaces. Using these tools we return to the homogeneous case with significantly weaker assumptions on $u_{0}$ in Section 7 . Section 8 is devoted to the inhomogeneous case of (1) when $u_{0}$ is identically zero.

2. Notations. In this paper we write $\mathbf{C}^{+}=\{\lambda \in \mathbf{C} ; \Re(\lambda)>0\}$ and $\mathbf{C}_{0}^{+}=\{\lambda \in \mathbf{C} ; \Re(\lambda) \geq 0\}$.

A function $\kappa \mapsto \varphi(\kappa)$ defined on some domain is denoted by $\varphi(\underline{\kappa})$. In particular $\underline{\kappa}$ denotes the function $\kappa \mapsto \kappa$.

The function $\mathbf{1}$ is defined by $\mathbf{1}(\kappa):=1$ for all $\kappa$.

The Laplace transform of a function $f$ is denoted by $\hat{f}$.

We use the notation $C_{0}[0, T]$ and $C_{0}^{\alpha}[0, T]$, where $\alpha>0$, for functions which are continuous respectively $\alpha$-Hölder continuous on $[0, T]$, and which are zero at zero.

3. Statement of the results. Throughout this paper we assume that the kernel $a$ satisfies the following hypothesis:

Hypothesis 3.1. The kernel $a:(0, \infty) \rightarrow \mathbf{R}$ is completely monotonic and such that $\int_{0}^{1} a(t) d t<\infty$ and $a(0+)=+\infty$. 
Definition 3.2. A solution to problem (1) is a function $u: \mathbf{R} \rightarrow \mathbf{R}$ such that

(i) $u$ is Borel measurable;

(ii) $\int_{-\infty}^{t} a(t-s)|u(s)| d s<\infty$ for $t \geq 0$;

(iii) The function $v:[0, \infty) \rightarrow \mathbf{R}$ defined by

$v(t):=\int_{-\infty}^{t} a(t-s) u(s) d s$ for $t \geq 0$ is absolutely continuous on $[0, T]$ for every $T>0$;

(iv) $u$ satisfies (1) almost everywhere.

Proposition 3.3. Problem (1) admits at most one solution.

Proof. Without loss of generality we assume that $u_{0}$ and $f$ are identically zero. Let $u$ be a solution to (1). Since $v$ is absolutely continuous, $v(0)=0$, and $(d / d t) v(t)=0$ for $t \geq 0$, almost everywhere, we have that $v$ is identically zero. It is a consequence of Titchmarsh's theorem, see [18, p. 166], that $u(t)=0$ for $t \geq 0$ almost everywhere. 口

The idea of this paper is to rewrite the homogeneous case of (1) with $f$ identically zero in an abstract setting

$$
\begin{aligned}
\frac{d}{d t} \psi(t) & =A \psi(t), \quad t>0, \\
\psi(0) & =\psi_{0},
\end{aligned}
$$

Therefore we take an arbitrary constant $\beta>0$ that will be fixed throughout the paper. We define the complex Hilbert space $H$ by

$$
\begin{array}{r}
H:=\{\varphi:[0, \infty) \longrightarrow \mathbf{C} \text { (equivalence class) } ; \quad \varphi \text { is Borel measurable } \\
\text { and } \left.\int_{[0, \infty)}|\varphi(\kappa)|^{2}(\kappa+\beta) \nu(d \kappa)<\infty\right\},
\end{array}
$$

endowed with the inner product

$$
\langle\varphi, \psi\rangle:=\int_{[0, \infty)} \varphi(\kappa) \overline{\psi(\kappa)}(\kappa+\beta) \nu(d \kappa), \quad \varphi, \psi \in H .
$$


We remark that $H$ is independent of $\beta$, and that for different $\beta \mathrm{s}$ the corresponding norms on $H$ are equivalent. We define the linear functional $J: D(J) \subseteq H \rightarrow \mathbf{C}$ by

(8)

$$
\begin{aligned}
& D(J):=\{\varphi \in H ; \text { there exists (a unique) } u \in \mathbf{C} \\
& \text { such that } u \mathbf{1}-\underline{\kappa} \varphi(\underline{\kappa}) \in H\}, \\
& J(\varphi):=u, \quad \varphi \in D(J) .
\end{aligned}
$$

We choose the letter $u$, because later it will appear that $u(t):=J(\psi(t))$ for $t>0$ is the solution to (1) with $f$ identically zero, where $\psi(t)$ is the solution to (6). Using $J$ we define the linear operator $A: D(A) \subseteq$ $H \rightarrow H$ by

$$
\begin{aligned}
D(A) & :=\left\{\psi \in D(J) ; \int_{[0, \infty)}(J(\psi)-\kappa \psi(\kappa)) \nu(d \kappa)=0\right\} \\
(A \psi)(\kappa) & :=J(\psi)-\kappa \psi(\kappa), \quad \psi \in D(A), \kappa \geq 0 .
\end{aligned}
$$

Theorem 3.4. The linear operator $A: D(A) \subseteq H \rightarrow H$ is the infinitesimal generator of a strongly continuous, positive, analytic semigroup $\{S(t)\}_{t \geq 0}$ on $H$ satisfying $\|S(t)\|_{\mathcal{L}(H)} \leq e^{(\beta t / 4)}$ for $t \geq 0$.

Hypothesis 3.5. The function $u_{0}:(-\infty, 0] \rightarrow \mathbf{R}$ is such that

(i) $u_{0}$ is Borel measurable;

(ii) There exist $M_{1}>0$ and $\omega>0$ such that $\left|u_{0}(t)\right| \leq M_{1} e^{\omega t}$ for

$$
t \leq 0
$$

(iii) There exist $M_{2}>0$ and $\delta>0$ such that $\left|u_{0}(0)-u_{0}(t)\right| \leq M_{2}|t|$ for $-\delta \leq t \leq 0$

(iv) $u_{0}(0) a(+\infty)+\int_{0}^{\infty}(-(d / d t) a(t))\left(u_{0}(0)-u_{0}(-t)\right) d t=0$.

Remark 3.6. The integral in Hypothesis 3.5 (iv) exists by Hypothesis 
3.5(ii) and (iii). Indeed, using Lemma 4.1 (ix), (iv), and (i), we have

$$
\begin{aligned}
\int_{0}^{\infty} & \left|\left(-\frac{d}{d t} a(t)\right)\left(u_{0}(0)-u_{0}(-t)\right)\right| d t \\
& \leq M_{2} \int_{0}^{\delta} t\left(-\frac{d}{d t} a(t)\right) d t+2 M_{1} \int_{\delta}^{\infty}-\frac{d}{d t} a(t) d t \\
& \leq-\underbrace{M_{2} \delta a(\delta)}_{\geq 0}+M_{2} \int_{0}^{\delta} a(t) d t-\underbrace{2 M_{1} a(+\infty)}_{\geq 0}+2 M_{1} a(\delta)<\infty .
\end{aligned}
$$

Remark 3.7. Hypothesis 3.5 does not assume any smoothness of $u_{0}$ except at zero.

Remark 3.8. If $u_{0}$ satisfies Hypothesis 3.5(i), (ii) and (iii), then

$$
\begin{aligned}
\left.\frac{d^{-}}{d t}\right|_{t=0} \int_{-\infty}^{t} a(t-s) u(s) d s= & u_{0}(0) a(+\infty) \\
& +\int_{0}^{\infty}\left(-\frac{d}{d t} a(t)\right)\left(u_{0}(0)-u_{0}(-t)\right) d t
\end{aligned}
$$

see [3] for a proof. Therefore, Hypothesis 3.5(iv) can be reformulated as

$$
\left.\frac{d^{-}}{d t}\right|_{t=0} \int_{-\infty}^{t} a(t-s) u(s) d s=0 .
$$

In addition, it follows from the proof of Lemma 5.1 that $\psi_{0}$ given by (3) belongs to $D(A)$ if and only if Hypothesis 3.5(iv) is satisfied.

Theorem 3.9. Let $u_{0}$ satisfy Hypothesis 3.5. Then problem (1) with $f$ identically zero admits a unique solution $u$. Moreover, $u$ is continuous on $[0, \infty)$, real analytic on $(0, \infty)$, and

$$
u(t)= \begin{cases}J\left(S(t) \psi_{0}\right) & t>0, \\ u_{0}(t), & t \leq 0,\end{cases}
$$

where $\psi_{0}$ is given by (3). 
Hypothesis 3.10. The kernel $a:(0, \infty) \rightarrow \mathbf{R}$ is such that there exist $0<\eta \leq \theta<1$ with

(i) $\int_{1}^{\infty} t^{-1-2 \eta}\left(1 /(\hat{a}(t))^{2}\right)(\hat{a}(t)+t(d / d t) \hat{a}(t)) d t<\infty$;

(ii) $\int_{1}^{\infty} t^{2 \theta-3}\left(1 /(\hat{a}(t))^{2}\right)(\hat{a}(t)+t(d / d t) \hat{a}(t)) d t<\infty$.

Remark 3.11. The following conditions imply that Hypothesis 3.10 holds:

(i) $\int_{1}^{\infty} t^{-1-2 \eta}(1 / \hat{a}(t)) d t<\infty$;

(ii) $\int_{1}^{\infty} t^{2 \theta-3}(1 / \hat{a}(t)) d t<\infty$.

Remark 3.12. Hypothesis 3.10(i) is equivalent to the fact that the restriction $\left.J\right|_{D(A)}$ may be extended continuously to a linear bounded functional from the interpolation space $D_{A}(\eta, 2)$ into $\mathbf{C}$ with the same $\eta$ as in Hypothesis 3.10, see Proposition 6.5.

Remark 3.13. Hypothesis 3.10(ii) is equivalent to the fact that the function $\pi \in H$ defined by

$$
\pi(\kappa):=\frac{1}{\beta \hat{a}(\beta)} \frac{1}{\kappa+\beta}, \quad \kappa \in[0, \infty)
$$

belongs to the interpolation space $D_{A}(\theta, 2)$ with the same $\theta$ as in Hypothesis 3.10, see Proposition 6.6.

Remark 3.14. Since $D(J)=D(A) \oplus$ span $\{\pi\}$, see Lemma 8.1, it follows that if $\pi$ belongs to $D_{A}(\theta, 2)$ for some $0<\theta<1$, then $D(J) \subseteq D_{A}(\alpha, 2)$ for every $0<\alpha \leq \theta$.

Theorem 3.15. Let $u_{0}$ satisfy Hypothesis 3.5 (i), (ii) and (iii). Let a satisfy Hypothesis 3.10. Then problem (1) with $f$ identically zero admits a unique solution $u$. Moreover, $u$ belongs to $C^{\theta-\eta}[0, \infty)$, is real analytic on $(0, \infty)$, and is given by (10). If in addition Hypothesis 3.5(iv) holds, then $u$ belongs to $C^{1-\eta}[0, \infty)$.

Remark 3.16. Let $u$ be the solution to (1) with $f$ identically zero under 
the conditions stated in Theorem 3.15. Then the following holds:

$$
\left(S(t) \psi_{0}\right)(\kappa)=\int_{0}^{\infty} e^{-\kappa s} u(t-s) d s, \quad t \geq 0, \kappa \geq 0 .
$$

Theorem 3.17. Let a satisfy Hypothesis 3.10 with $0<\eta<\theta<1$. Let $f$ belong to $L^{p}[0, T]$ for every $T>0$ where $1 \leq p<\infty$. Then problem (1) with $u_{0}$ identically zero admits a unique solution $u$ and

$$
u(t)= \begin{cases}J\left(\int_{0}^{t}(\beta I-A) S(t-s) f(s) \pi d s\right) & t>0, \\ 0 & t \leq 0,\end{cases}
$$

where the integral is a Bochner integral in $D_{A}(\eta, 2)$ and $\pi$ is given by (11). Moreover, the following holds:

(i) If $p=1$, then $u$ belongs to $L^{q}[0, T]$ for every $1 \leq q<\frac{1}{1-(\theta-\eta)}$ and $T>0$;

(ii) If $1<p<1 /(\theta-\eta)$, then $u$ belongs to $L^{q}[0, T]$ for every $1 \leq q \leq \frac{p}{1-(\theta-\eta) p}$ and $T>0$

(iii) If $p=1 /(\theta-\eta)$, then $u$ belongs to $L^{q}[0, T]$ for every $1 \leq q<\infty$ and $T>0$

(iv) If $1 /(\theta-\eta)<p<\infty$, then $u$ belongs to $C_{0}^{\theta-\eta-(1 / p)}[0, \infty)$.

More regularity results for problem (1) can be obtained using the machinery of analytic semigroups. An example is the following theorem, for a proof of which we refer to $[\mathbf{3}]$.

Theorem 3.18. Let a satisfy Hypothesis 3.10 with $0<\eta<\theta<1$. Let $f$ belong to $C_{0}^{\gamma}[0, \infty)$ where $0<\gamma<1$ is such that $\gamma \neq 1-(\theta-\eta)$. Then problem (1) with $u_{0}$ identically zero admits a unique solution $u$. Moreover, $u$ is given by (12) and belongs to $C_{0}^{\gamma+\theta-\eta}[0, \infty)$.

Example 3.19. Let the kernel $a:(0, \infty) \rightarrow \mathbf{R}$ be given by

$$
a(t):=e^{-\omega t} g_{1-\alpha}(t), \quad t>0,
$$


with $\omega \geq 0$ and $0<\alpha<1$, where the function $g_{\rho}:(0, \infty) \rightarrow \mathbf{R}$ with $\rho>0$ is defined by

$$
g_{\rho}(t):=\frac{t^{\rho-1}}{\Gamma(\rho)}, \quad t>0
$$

By straightforward calculation Hypothesis 3.10(i) holds if and only if $\eta>(1-\alpha) / 2$, and Hypothesis 3.10(ii) holds if and only if $\theta<(1+\alpha) / 2$. Since $0<\alpha<1$ we can choose $0<\eta<\theta<1$ such that $(1-\alpha) / 2<$ $\eta<\theta<(1+\alpha) / 2$. Let $\omega=0$ and let $f$ belong to $L^{p}[0, T]$ for every $T>0$ where $1 \leq p<\infty$. Since we have that for $0<\rho<1$

$$
\int_{0}^{t} g_{\rho}(t-s) g_{1-\rho}(s) d s=1, \quad t>0
$$

it is easy to verify that $u: \mathbf{R} \rightarrow \mathbf{R}$ given by

$$
u(t)= \begin{cases}\int_{0}^{t} g_{\alpha}(t-s) f(s) d s & t>0 \\ 0 & t \leq 0\end{cases}
$$

is the unique solution to (1) with $u_{0}$ identically zero. Moreover, it follows from Proposition A.1 that

(i) If $p=1$, then $u$ belongs to $L^{q}[0, T]$ for every $1 \leq q<1 /(1-\alpha)$ and $T>0$;

(ii) If $1<p<1 / \alpha$, then $u$ belongs to $L^{q}[0, T]$ for every $1 \leq q \leq p /(1-\alpha p)$ and $T>0 ;$

(iii) If $p=(1 / \alpha)$, then $u$ belongs to $L^{q}[0, T]$ for every $1 \leq q<\infty$ and $T>0$;

(iv) If $(1 / \alpha)<p<\infty$, then $u$ belongs to $C_{0}^{\alpha-\frac{1}{p}}[0, \infty)$.

4. The semigroup setting. We recall that the complex Hilbert space $H$, the linear functional $J: D(J) \subseteq H \rightarrow \mathbf{C}$, and the linear operator $A: D(A) \subseteq H \rightarrow H$ are defined by respectively (7), (8) and (9). The main result of this section is Theorem 3.4. Before we are in a position to prove this theorem we need some preliminaries. We start 
with listing properties of the kernel $a$ and the measure $\nu$, which we shall use throughout the paper.

\section{Lemma 4.1.}

(i) $\int_{0}^{T} a(t) d t<\infty$ for every $T>0$;

(ii) $(-1)^{n} \frac{d^{n}}{d t^{n}} a(t)=\int_{[0, \infty)} e^{-\kappa t} \kappa^{n} \nu(d \kappa)$ for $t>0$ and $n=0,1,2, \ldots$;

(iii) $(-1)^{n} \frac{d^{n}}{d t^{n}} a(0+)=\int_{[0, \infty)} \kappa^{n} \nu(d \kappa)=+\infty$ for $n=0,1,2, \ldots$;

(iv) $0 \leq \lim _{t \rightarrow \infty} a(t)<\infty$;

(v) $\lim _{t \rightarrow \infty}(-1)^{n} \frac{d^{n}}{d t^{n}} a(t)=0$ for $n=1,2, \ldots$;

(vi) If $\lambda \in \mathbf{C} \backslash\{0\}$ is such that $\lambda=\mu+i \omega$ with $\mu \geq 0$ and $\omega \in \mathbf{R}$, then $\hat{a}(\lambda)=\int_{[0, \infty)} \frac{1}{\kappa+\lambda} \nu(d \kappa)=\int_{[0, \infty)} \frac{\kappa+\mu-i \omega}{(\kappa+\mu)^{2}+\omega^{2}} \nu(d \kappa)<\infty$;

(vii) $\int_{[0, T]} \nu(d \kappa)<\infty$ for $T>0$;

(viii) $\int_{[\delta, \infty)}(1 / \kappa) \nu(d \kappa)<\infty$ for $\delta>0$;

(ix) $\lim _{t \downarrow 0} t a(t)=0$;

(x) $\lim _{t \downarrow 0} t^{2} \frac{d}{d t} a(t)=0$;

(xi) $-\frac{d}{d \lambda} \hat{a}(\lambda)=\int_{[0, \infty)} \frac{1}{(\kappa+\lambda)^{2}} \nu(d \kappa)$ for $\lambda>0$;

(xii) $\int_{[0, \infty)} \frac{\kappa+c}{(\kappa+\lambda)^{2}} \nu(d \kappa)=\hat{a}(\lambda)+(\lambda-c) \frac{d}{d \lambda} \hat{a}(\lambda)$ for $\lambda>0$ and $c \in \mathbf{R}$; 


$$
\begin{aligned}
& \begin{aligned}
\text { (xiii) } \int_{[0, \infty)} \frac{\kappa^{2}}{(\kappa+\lambda)^{2}} \frac{1}{\kappa+\beta} \nu(d \kappa)= & \left(1-\frac{\beta^{2}}{(\lambda-\beta)^{2}}\right) \hat{a}(\lambda)+\frac{\lambda^{2}}{\lambda-\beta} \frac{d}{d \lambda} \hat{a}(\lambda) \\
& +\frac{\beta^{2}}{(\lambda-\beta)^{2}} \hat{a}(\beta) \text { for } \lambda>0 ;
\end{aligned} \\
& \left(\text { xiv) }-\lambda \frac{d}{d \lambda} \hat{a}(\lambda) \leq \hat{a}(\lambda) \text { for } \lambda>0 ;\right. \\
& (\text { xv) } \lambda \hat{a}(\lambda) \geq c \hat{a}(c) \text { for } \lambda>c>0 ; \\
& \left(\text { xvi) } 0 \leq \hat{a}(\beta)-\hat{a}(\lambda) \leq \frac{\lambda-\beta}{\beta} \hat{a}(\lambda) \text { for } \lambda>\beta .\right.
\end{aligned}
$$

Proof. For property (ii) and the first equality in property (iii) we refer to [13, p. 90]. The second equality in property (iii) follows from

$$
a(t)=a(T)+\int_{t}^{T}-\frac{d}{d t} a(s) d s \leq a(T)-(T-t) \frac{d}{d t} a(t), \quad 0<t<T,
$$

which implies that if $(-d / d t) a(0+)<\infty$, then $a(0+)<\infty$, contradicting the assumption that $a(0+)=+\infty$. Property (v) follows from

$$
a(T)=a(t)+\int_{t}^{T} \frac{d}{d t} a(s) d s \leq a(t)+(T-t) \frac{d}{d t} a(T), \quad 0<t<T,
$$

which implies that if $\lim _{T \rightarrow \infty}-(d / d t) a(T)>0$, then $\lim _{T \rightarrow \infty} a(T)<0$, contradicting Property (iv). By straightforward calculation it follows that property (vi) holds for $\lambda \in \mathbf{C}^{+}$. Properties (vii) and (viii) follow from respectively

$$
\begin{aligned}
\int_{[0, T]} \nu(d \kappa) & \leq \int_{[0, T]} \frac{T+\beta}{\kappa+\beta} \nu(d \kappa) \\
& \leq(T+\beta) \int_{[0, \infty)} \frac{1}{\kappa+\beta} \nu(d \kappa), \int_{[\delta, \infty)} \frac{1}{\kappa} \nu(d \kappa) \leq \frac{\beta+\delta}{\delta} \\
\int_{[\delta, \infty)} \frac{1}{\kappa+\beta} \nu(d \kappa) & \leq \frac{\beta+\delta}{\delta} \int_{[0, \infty)} \frac{1}{\kappa+\beta} \nu(d \kappa) .
\end{aligned}
$$

For $\lambda \in \mathbf{C}$ such that $\lambda=i \omega$ with $\omega \neq 0$, property (vi) follows from Lebesgue's dominated convergence theorem for

$$
\lim _{\mu \downarrow 0} \int_{[0, \infty)} \frac{1}{\kappa+\mu+i \omega} \nu(d \kappa)=\int_{[0, \infty)} \frac{1}{\kappa+i \omega} \nu(d \kappa)
$$


using Lemma 4.1(vii) and (viii) and

$$
\begin{aligned}
\int_{[0, \infty)} \frac{1}{|\kappa+i \omega|} \nu(d \kappa) & \leq \int_{[0,1]} \frac{1}{\sqrt{\kappa^{2}+\omega^{2}}} \nu(d \kappa)+\int_{[1, \infty)} \frac{1}{\sqrt{\kappa^{2}+\omega^{2}}} \nu(d \kappa) \\
& \leq \frac{1}{|\omega|} \int_{[0,1]} \nu(d \kappa)+\int_{[1, \infty)} \frac{1}{\kappa} \nu(d \kappa)<\infty
\end{aligned}
$$

Property (ix), and analogously Property (x), follows from

$$
t a(t)=\int_{[0, \infty)} t e^{-\kappa t} \nu(d \kappa)=\int_{[0, \infty)}\left(\kappa t e^{-\kappa t}+\beta t e^{-\kappa t}\right) \frac{1}{\kappa+\beta} \nu(d \kappa)
$$

for $t>0$ and Lebesgue's dominated convergence theorem. Property (xv) is a consequence of the fact that $t \mapsto \frac{t}{\kappa+t}$ is a positive, monotonically increasing function for $\kappa \geq 0$. The properties which have not been mentioned follow by straightforward calculation.

The next two lemmas give properties of the Hilbert space $H$. The first lemma can be proved using the Cauchy-Schwarz inequality and Lemma 4.1(vi).

Lemma 4.2. The Hilbert space $H$ is continuously embedded in $L^{1}([0, \infty), d \nu)$ with $\|\varphi\|_{L^{1}([0, \infty), d \nu)} \leq \sqrt{\hat{a}(\beta)}\|\varphi\|$ for $\varphi \in H$.

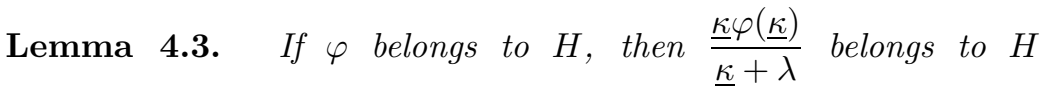
for $\lambda \in \mathbf{C}_{0}^{+}$and $\left\|\frac{\underline{\kappa} \varphi(\underline{\kappa})}{\kappa+\lambda}\right\| \leq\|\varphi\|$. If in addition $\lambda \neq 0$, then $\frac{1}{\underline{\kappa}+\lambda}$ and $\frac{\varphi(\underline{\kappa})}{\underline{\kappa}+\lambda}$ belong to $H$ and $\left\|\frac{\varphi(\underline{\kappa})}{\underline{\kappa}+\lambda}\right\| \leq \frac{1}{|\lambda|}\|\varphi\|$. Moreover, $\left\|\frac{1}{\underline{\kappa}+\lambda}\right\| \leq 2 \sqrt{\Re\left(\hat{a}\left(\frac{\beta}{4}+i \omega\right)\right)}$ for $\lambda=\mu+i \omega$ with $\mu \geq \frac{\beta}{4}$ and $\omega \in \mathbf{R}$.

Proof. We only prove the last estimate. The rest follows by straightforward calculation and Lemma 4.1(xii). Let $\lambda \in \mathbf{C}^{+}$be such that $\lambda=\mu+i \omega$ with $\mu \geq(\beta / 4)$ and $\omega \in \mathbf{R}$. Then we have using 
Lemma 4.1(vi).

$$
\begin{aligned}
\int_{[0, \infty)}\left|\frac{1}{\kappa+\lambda}\right|^{2}(\kappa+\beta) \nu(d \kappa) & \leq \int_{[0, \infty)} \frac{\kappa+\beta}{\left(\kappa+\frac{\beta}{4}\right)^{2}+\omega^{2}} \nu(d \kappa) \\
& \leq 4 \int_{[0, \infty)} \frac{\kappa+(\beta / 4)}{\left(\kappa+\frac{\beta}{4}\right)^{2}+\omega^{2}} \nu(d \kappa) \\
& =4 \Re\left(\hat{a}\left(\frac{\beta}{4}+i \omega\right)\right) .
\end{aligned}
$$

The following lemma implies that the linear functional $J: D(J) \subseteq$ $H \rightarrow \mathbf{C}$ is well defined. The proof of the lemma is straightforward, using the assumption that $a(0+)=+\infty$ and Lemma 4(iii).

Lemma 4.4. For every $\varphi \in H$ there exists at most one constant $u \in \mathbf{C}$ such that $u \mathbf{1}-\underline{\kappa} \varphi(\underline{\kappa})$ belongs to $H$.

It is a consequence of Lemma 4.2 that the linear operator $A: D(A) \subseteq$ $H \rightarrow H$ is well-defined. Furthermore, we have the next proposition.

Proposition 4.5. The linear operator $A-(\beta / 4) I: D(A) \subseteq H \rightarrow H$ is dissipative in $(H,\langle\cdot, \cdot\rangle)$.

Proof. Let $\psi$ belong to $D(A)$. Using the definition of $D(A)$, Lemma 4.1(vi), and Lemma 4.2, we have

$$
\begin{aligned}
\Re\langle A \psi, \psi\rangle= & \Re \int_{[0, \infty)}(J(\psi)-\kappa \psi(\kappa) \overline{\psi(\kappa)}(\kappa+\beta) \nu(d \kappa) \\
& -\overline{J(\psi)} \underbrace{\int_{[0, \infty)}(J(\psi)-\kappa \psi(\kappa)) \nu(d \kappa)}_{=0} \\
= & \Re \int_{[0, \infty)}(J(\psi)-\kappa \psi(\kappa))(\overline{\psi(\kappa)}(\kappa+\beta)-\overline{J(\psi)}) \nu(d \kappa) \\
= & \Re \int_{[0, \infty)} \frac{\kappa}{\kappa+\beta}(J(\psi)-(\kappa+\beta) \psi(\kappa))(\overline{\psi(\kappa)}(\kappa+\beta)-\overline{J(\psi)}) \nu(d \kappa) \\
& +\beta \Re \int_{[0, \infty)} J(\psi) \overline{\psi(\kappa)} \nu(d \kappa)-\beta|J(\psi)|^{2} \int_{[0, \infty)} \frac{1}{\kappa+\beta} \nu(d \kappa)
\end{aligned}
$$




$$
\begin{aligned}
\leq & -\int_{[0, \infty)} \underbrace{\frac{\kappa}{\kappa+\beta}|J(\psi)-(\kappa+\beta) \psi(\kappa)|^{2}}_{\geq 0} \nu(d \kappa) \\
& +\beta|J(\psi)| \int_{[0, \infty)}|\psi(\kappa)| \nu(d \kappa)-\beta \hat{a}(\beta)|J(\psi)|^{2} \\
\leq & \beta \sqrt{\hat{a}(\beta)}|J(\psi)|\|\psi\|-\beta \hat{a}(\beta)|J(\psi)|^{2} \\
= & -\beta \underbrace{(\sqrt{\hat{a}(\beta)}|J(\psi)|-\|\psi\| / 2)^{2}}_{\geq 0}+\frac{\beta}{4}\|\psi\|^{2} \leq \frac{\beta}{4}\|\psi\|^{2}
\end{aligned}
$$

This implies that $\Re\langle(A-(\beta / 4) I) \psi, \psi\rangle \leq 0$, which shows the dissipativity of $A-\beta I / 4$.

It follows from the next lemma that the linear operator $\lambda I-A$ : $D(A) \rightarrow H$ is onto for $\lambda \in \mathbf{C}_{0}^{+} \backslash\{0\}$.

Lemma 4.6. Let $\lambda \in \mathbf{C}_{0}^{+} \backslash\{0\}$ and let $\varphi$ belong to $H$. Let $u \in \mathbf{C}$ be given by

$$
u:=\frac{1}{\lambda \hat{a}(\lambda)} \int_{[0, \infty)} \frac{\kappa \varphi(\kappa)}{\kappa+\lambda} \nu(d \kappa) .
$$

Let $\psi:[0, \infty) \rightarrow \mathbf{C}$ be defined by

$$
\psi(\kappa):=\frac{\varphi(\kappa)+u}{\kappa+\lambda}, \quad \kappa \in[0, \infty)
$$

Then $u$ is well-defined, $\psi$ belongs to $D(A), J(\psi)=u$, and $(\lambda I-A) \psi=$ $\varphi$. Moreover, if $\lambda=\mu+i \omega$ with $\mu \geq(\beta / 4)$ and $\omega \in \mathbf{R}$, then

$$
|u| \leq 2 \frac{1}{|\lambda|} \frac{1}{|\hat{a}(\lambda)|} \sqrt{\Re\left(\hat{a}\left(\left(\frac{\beta}{4}+i \omega\right)\right)\right.}\|\varphi\| .
$$

Proof. The constant $u$ is well-defined by Lemmas 4.3 and 4.2. Since $\frac{\varphi(\underline{\kappa})}{\underline{\kappa}+\lambda}$ and $\frac{1}{\underline{\kappa}+\lambda}$ belong to $H$ by Lemma 4.3 , it follows that $\psi$ belongs to $H$. Furthermore, using the definition of $\psi$ we have that 
$u \mathbf{1}-\underline{\kappa} \psi(\underline{\kappa})=\lambda \psi-\varphi$ belongs to $H$ and hence, $\psi$ belongs to $D(J)$ with $J(\psi)=u$. By straightforward calculation it follows that $\psi$ belongs to $D(A)$ and that $(\lambda I-A) \psi=\varphi$. Finally, if $\lambda=\mu+i \omega$ with $\mu \geq(\beta / 4)$ and $\omega \in \mathbf{R}$, then we have using the Cauchy-Schwarz inequality and Lemma 4.3,

$$
\begin{aligned}
|u| & \leq \frac{1}{|\lambda|} \frac{1}{|\hat{a}(\lambda)|} \int_{[0, \infty)}\left|\frac{\varphi(\kappa)}{\kappa+\lambda}\right|(\kappa+\beta) \nu(d \kappa) \\
& \leq \frac{1}{|\lambda|} \frac{1}{|\hat{a}(\lambda)|}\left\|\frac{1}{\underline{\kappa}+\lambda}\right\|\|\varphi\| \\
& \leq 2 \frac{1}{|\lambda|} \frac{1}{|\hat{a}(\lambda)|} \sqrt{\Re\left(\hat{a}\left(\frac{\beta}{4}+i \omega\right)\right)}\|\varphi\| .
\end{aligned}
$$

The following proposition characterizes the resolvent of $A$.

Proposition 4.7. If $\lambda \in \mathbf{C}_{0}^{+} \backslash\{0\}$, then the linear operator $\lambda I-A$ : $D(A) \rightarrow H$ is bijective and

$$
(\lambda I-A)^{-1} \varphi=\frac{\varphi(\underline{\kappa})+u}{\underline{\kappa}+\lambda}, \quad \varphi \in H
$$

where $u$ is given by (14). Moreover, $J\left((\lambda I-A)^{-1} \varphi\right)=u$. If in addition $\lambda=\mu+i \omega$ with $\mu \geq \frac{\beta}{4}$ and $\omega \in \mathbf{R}$, then $0 \in \rho(\lambda I-A)$ and

$$
\left\|(\lambda I-A)^{-1}\right\|_{\mathcal{L}(H)} \leq\left(1+4 \frac{\Re(\hat{a}((\beta / 4)+i \omega))}{|\hat{a}(\lambda)|}\right) \frac{1}{|\lambda|} .
$$

Proof. To show that $\lambda I-A: D(A) \rightarrow H$ is one-to-one for $\lambda \in$ $\mathbf{C}_{0}^{+} \backslash\{0\}$, let $\psi \in D(A)$ be such that $(\lambda I-A) \psi=0$. This implies that $\psi=\frac{J(\psi)}{\underline{\kappa}+\lambda}$, so that by definition of $A$, Lemma $4.1(\mathrm{vi})$, and some straightforward calculation

$$
0=\int_{[0, \infty)}(J(\psi)-\kappa \psi(\kappa)) \nu(d \kappa)=J(\psi) \lambda \hat{a}(\lambda)
$$


Therefore, $J(\psi)=0$ and hence, $\psi=0$.

Since the proposition is a consequence of Lemma 4.6, we only have to prove that the linear operator $(\lambda I-A)^{-1}: H \rightarrow H$ is bounded for $\lambda=\mu+i \omega$ with $\mu \geq \frac{\beta}{4}$ and $\omega \in \mathbf{R}$. Let $\varphi$ belong to $H$ and let $u \in \mathbf{C}$ be given by (14). Using Lemmas 4.3 and 4.6 we have

$$
\begin{aligned}
\left\|(\lambda I-A)^{-1} \varphi\right\| \leq & \left\|\frac{\varphi(\underline{\kappa})}{\underline{\kappa}+\lambda}\right\|+|u|\left\|\frac{1}{\underline{\kappa}+\lambda}\right\| \\
\leq & \frac{1}{|\lambda|}\|\varphi\|+2 \frac{1}{|\lambda|} \frac{1}{|\hat{a}(\lambda)|} \sqrt{\Re(\hat{a}((\beta / 4)+i \omega))}\|\varphi\| \\
& \cdot 2 \sqrt{\Re(\hat{a}((\beta / 4)+i \omega))} \\
= & \left(1+4 \frac{\Re(\hat{a}((\beta / 4)+i \omega))}{|\hat{a}(\lambda)|}\right) \frac{1}{|\lambda|}\|\varphi\| .
\end{aligned}
$$

We are now in a position to prove Theorem 3.4.

Proof. It is a consequence of Proposition 4.5 and Lemma 4.6 that the linear operator $A-\frac{\beta}{4} I: D(A) \subseteq H \rightarrow H$ is $m$-dissipative. Since $H$ is a reflexive space, $D(A)$ is dense in $H$, see $[\mathbf{1 2}$, p. 16]. From the Lumer-Phillips theorem, see [12, p. 14], it follows that $A-\frac{\beta}{4} I$ is the infinitesimal generator of a strongly continuous semigroup of contractions on $H$. Now we show that this semigroup is analytic. It is sufficient to show that there exists $K>0$ such that for every $\omega \neq 0$, $\left\|\left(i \omega I-\left(A-\frac{\beta}{4} I\right)\right)^{-1}\right\|_{\mathcal{L}(H)} \leq \frac{K}{|\omega|}$, see [4, p. 123]. Let $\omega \neq 0$. By applying Proposition 4.7 with $\lambda=(\beta / 4)+i \omega$ we have

$$
\begin{aligned}
\|(i \omega I-( & -(\beta / 4) I))^{-1} \|_{\mathcal{L}(H)} \\
& =\left\|\left(\left(\frac{\beta}{4}+i \omega\right) I-A\right)^{-1}\right\|_{\mathcal{L}(H)} \\
& \leq\left(1+4 \frac{\Re(\hat{a}((\beta / 4)+i \omega))}{|\hat{a}((\beta / 4)+i \omega)|}\right) \frac{1}{|(\beta / 4)+i \omega|} \\
& \leq(1+4 \cdot 1) \frac{1}{|\omega|}=\frac{5}{|\omega|} .
\end{aligned}
$$


Finally it follows that $A$ is the infinitesimal generator of a strongly continuous, analytic semigroup $\{S(t)\}_{t \geq 0}$ on $H$ satisfying $\|S(t)\|_{\mathcal{L}(H)} \leq$ $e^{(\beta / 4) t}$ for $t \geq 0$, see $[\mathbf{1 2}$, pp. 76, 80]. The positivity of the resolvent implies the positivity of the semigroup.

The following lemma is a consequence of Lemma 4.6 and will be used throughout the rest of the paper.

Lemma 4.8. The restriction $\left.J\right|_{D(A)}: D(A) \rightarrow \mathbf{C}$ is bounded with respect to the graph norm of $A$.

Proof. Let $\psi$ belong to $D(A)$. We define the function $\varphi \in H$ by $\varphi:=(\beta I-A) \psi$. By Lemma 4.6 we have

$$
J(\psi)=\frac{1}{\beta \hat{a}(\beta)} \int_{[0, \infty)} \frac{\kappa \psi(\kappa)}{\kappa+\beta} \nu(d \kappa) .
$$

Using the Cauchy-Schwarz inequality, Lemma 4.1(vi), and the norm $\|(\beta I-A) \cdot\|$ on $D(A)$, which is equivalent to the graph norm of $A$, it follows that

$$
\begin{aligned}
|J(\psi)| & \leq \frac{1}{\beta \hat{a}(\beta)}\left(\int_{[0, \infty)} \frac{\kappa}{(\kappa+\beta)^{2}} \nu(d \kappa)\right)^{1 / 2}\left(\int_{[0, \infty)}|\varphi(\kappa)|^{2} \kappa \nu(d \kappa)\right)^{1 / 2} \\
& \leq \frac{1}{\beta \hat{a}(\beta)} \sqrt{\hat{a}(\beta)}\|\varphi\| \\
& =\frac{1}{\beta \sqrt{\hat{a}(\beta)}}\|(\beta I-A) \psi\| .
\end{aligned}
$$

In Section 6 we need the adjoint operator $A^{*}$ of $A$. The next lemma gives a characterization of $A^{*}$.

Lemma 4.9. The adjoint operator $A^{*}: D\left(A^{*}\right) \subseteq H \rightarrow H$ is given by

$$
\begin{gathered}
D\left(A^{*}\right)=\left\{\sigma \in D(J) ; \int_{[0, \infty)}(J(\sigma)-(\kappa+\beta) \sigma(\kappa)) \nu(d \kappa)=0\right\} \\
\left(A^{*} \sigma\right)(\kappa)=J(\sigma) \frac{\kappa}{\kappa+\beta}-\kappa \sigma(\kappa), \quad \sigma \in D\left(A^{*}\right), \kappa \geq 0
\end{gathered}
$$


Proof. Let $\sigma \in H$. By definition of the adjoint operator $A^{*}, \sigma$ belongs to $D\left(A^{*}\right)=D\left(\beta I-A^{*}\right)$ if there exists $\tau \in H$ such that $\langle(\beta I-A) \psi, \sigma\rangle=\langle\psi, \tau\rangle$ for all $\psi \in D(A)$, or equivalently, such that

$$
\langle\varphi, \sigma\rangle=\left\langle(\beta I-A)^{-1} \varphi, \tau\right\rangle
$$

for all $\varphi \in H$. Let $\varphi \in H$ be arbitrary and let $u \in \mathbf{C}$ be given by (14). Using Proposition 4.7, (16) holds if and only if

$$
\int_{[0, \infty)} \varphi(\kappa) \overline{\sigma(\kappa)}(\kappa+\beta) \nu(d \kappa)=\int_{[0, \infty)}(\varphi(\kappa)+u) \overline{\tau(\kappa)} \nu(d \kappa)
$$

if and only if

$$
\begin{aligned}
\int_{[0, \infty)} & \left(\overline{\sigma(\kappa)}-\frac{\overline{\tau(\kappa)}}{\kappa+\beta}\right) \varphi(\kappa)(\kappa+\beta) \nu(d \kappa) \\
& =\frac{1}{\beta \hat{a}(\beta)}\left(\int_{[0, \infty)} \overline{\tau(\kappa)} \nu(d \kappa)\right) \int_{[0, \infty)} \frac{\kappa}{(\kappa+\beta)^{2}} \varphi(\kappa)(\kappa+\beta) \nu(d \kappa)
\end{aligned}
$$

if and only if

$$
\left\langle\varphi, \sigma-\frac{\tau(\underline{\kappa})}{\underline{\kappa}+\beta}-\frac{1}{\beta \hat{a}(\beta)}\left(\int_{[0, \infty)} \tau(\kappa) \nu(d \kappa)\right) \frac{\underline{\kappa}}{(\underline{\kappa}+\beta)^{2}}\right\rangle=0 .
$$

Since $\varphi \in H$ is arbitrary, this holds if and only if

$$
\tau=(\underline{\kappa}+\beta) \sigma(\underline{\kappa})-K \frac{\underline{\kappa}}{\underline{\kappa}+\beta},
$$

where, using Lemma 4.1 (vi), $K \in \mathbf{C}$ is such that

$$
\begin{aligned}
K & =\frac{1}{\beta \hat{a}(\beta)} \int_{[0, \infty)} \tau(\kappa) \nu(d \kappa) \\
& =\frac{1}{\beta \hat{a}(\beta)} \int_{[0, \infty)}((\kappa+\beta) \sigma(\kappa)-K) \nu(d \kappa)+K,
\end{aligned}
$$

or equivalently, where $K \in \mathbf{C}$ satisfies

$$
\int_{[0, \infty)}(K-(\kappa+\beta) \sigma(\kappa)) \nu(d \kappa)=0
$$


From (17) and Lemma 4.3 it follows that $\tau$ belongs to $H$ if and only if $\underline{\kappa} \sigma(\underline{\kappa})-K 1$ belongs to $H$. We conclude that $\sigma$ belongs to $D\left(A^{*}\right)$ if and only if there exists $K \in \mathbf{C}$ such that $\underline{\kappa} \sigma(\underline{\kappa})-K \mathbf{1}$ belongs to $H$ and (18) holds, that is, $\sigma$ belongs to $D(J)$ and satisfies

$$
\int_{[0, \infty)}(J(\sigma)-(\kappa+\beta) \sigma(\kappa)) \nu(d \kappa)=0
$$

Finally, (17) implies that $\left(\beta I-A^{*}\right) \sigma=(\underline{\kappa}+\beta) \sigma(\underline{\kappa})-J(\sigma) \frac{\underline{\kappa}}{\underline{\kappa}+\beta}$ and hence, (15) holds.

Since the linear operator $A-(\beta / 4) I: D(A) \subseteq H \rightarrow H$ is mdissipative in $(H,\langle\cdot, \cdot\rangle)$, it is well-known that the linear operator $A^{*}-$ $(\beta / 4) I: D\left(A^{*}\right) \rightarrow H$ is $m$-dissipative in $(H,\langle\cdot, \cdot\rangle)$ as well. Furthermore, since $0 \in \rho(\lambda I-A)$ for $\lambda \in \mathbf{C}$ with $\Re(\lambda) \geq(\beta / 4)$ by Proposition 4.7, we also have $0 \in \rho\left(\lambda I-A^{*}\right)$ and $\left(\lambda I-A^{*}\right)^{-1}=\left((\lambda I-A)^{-1}\right)^{*}$ for $\lambda \in \mathbf{C}$ with $\Re(\lambda) \geq(\beta / 4)$. See [12, pp. 38, 41].

The following proposition characterizes the resolvent of $A^{*}$.

Proposition 4.10. If $\lambda>0$, then $\left(\lambda I-A^{*}\right)^{-1}: H \rightarrow H$ is given by

$$
\left(\lambda I-A^{*}\right)^{-1} \tau=\frac{1}{\underline{\kappa}+\lambda}\left(\tau+p \frac{\underline{\kappa}}{\underline{\kappa}+\beta}\right), \quad \tau \in H,
$$

where

$$
p=\frac{1}{\lambda \hat{a}(\lambda)} \int_{[0, \infty)} \frac{\kappa+\beta}{\kappa+\lambda} \tau(\kappa) \nu(d \kappa) .
$$

Moreover, $J\left(\left(\lambda I-A^{*}\right)^{-1} \tau\right)=p$.

Proof. Let $\lambda>0$ and let $\tau$ belong to $H$. Let $\sigma:[0, \infty) \rightarrow \mathbf{C}$ be defined by

$$
\sigma(\kappa):=\frac{1}{\underline{\kappa}+\lambda}\left(\tau+p \frac{\underline{\kappa}}{\underline{\kappa}+\beta}\right), \quad \kappa \geq 0 .
$$

Using Lemma 4.3, the constant $p$ is well-defined by the inner product of $\frac{1}{\underline{\kappa}+\lambda}$ and $\overline{\tau(\underline{\kappa})}$. By Lemma $4.3, \sigma$ belongs to $H$. It is a consequence of 
the definition of $\sigma$ and Lemma 4.3 that $p \mathbf{1}-\underline{\kappa} \sigma(\underline{\kappa})=\lambda \sigma-\tau+p \frac{\beta}{\underline{\kappa}+\lambda}$ belongs to $H$. Hence, $\sigma$ belongs to $D(J)$ with $J(\sigma)=p$. By straightforward calculation it follows that $\sigma$ belongs to $D\left(A^{*}\right)$ and $\left(\lambda I-A^{*}\right) \sigma=\tau$.

5. The homogeneous case $\mathbf{I}$. The goal of this section is to prove Theorem 3.9. To this end we consider the homogeneous problem (1) with $f$ identically zero and $u_{0}$ satisfying Hypothesis 3.5. We first study the following homogeneous abstract Cauchy problem in $H$ :

$$
\begin{aligned}
\frac{d}{d t} \psi(t) & =A \psi(t), \quad t>0, \\
\psi(0) & =\psi_{0},
\end{aligned}
$$

where $\psi_{0}:[0, \infty) \rightarrow \mathbf{R}$ is given by (3). By straightforward calculation using Hypothesis 3.5(ii) and Lemma 4.1(xii) it follows that $\psi_{0}$ is welldefined and belongs to $H$. Furthermore, we have the next lemma.

Lemma 5.1. The function $\psi_{0}$ belongs to $D(A)$ and $J\left(\psi_{0}\right)=u_{0}(0)$.

Proof. Let $u_{1}, u_{2}:(-\infty, 0] \rightarrow \mathbf{R}$ and $\psi_{1}, \psi_{2}:[0, \infty) \rightarrow \mathbf{R}$ be defined by respectively $u_{1}(t):=u_{0}(0) e^{\omega t}$ and $u_{2}(t):=u_{0}(t)-u_{0}(0) e^{\omega t}$ for $t \leq 0$ and

$$
\psi_{j}(\kappa):=\int_{0}^{\infty} e^{-\kappa s} u_{j}(-s) d s, \quad \kappa \geq 0, \quad j=1,2 .
$$

It is easily observed that $u_{1}$ and $u_{2}$ satisfy Hypothesis 3.5 (i), (ii), and (iii), and hence, that $\psi_{1}$ and $\psi_{2}$ are well-defined. Note that $u_{0}=u_{1}+u_{2}$ and $\psi_{0}=\psi_{1}+\psi_{2}$. It follows from straightforward calculation that $\psi_{1}(\kappa)=\frac{u_{0}(0)}{\kappa+\omega}$ for $\kappa \geq 0$, so that $\psi_{1}$ belongs to $H$ by Lemma 4.3 , and $\psi_{1}$ belongs to $D(J)$ with $J\left(\psi_{1}\right)=u_{0}(0)$ using Lemma 4.1(xii). Now we show that $\psi_{2}$ belongs to $D(J)$ with $J\left(\psi_{2}\right)=0$. Since $u_{2}$ satisfies Hypothesis 3.5(ii) and (iii), let $M_{1}^{\prime}>0$ and $M_{2}^{\prime}>0$ be such that $\left|u_{2}(t)\right| \leq M_{1}^{\prime} e^{\omega t}$ for $t \leq 0$ and $\left|u_{2}(t)\right|=\left|u_{2}(0)-u_{2}(t)\right| \leq M_{2}^{\prime}|t|$ for $-\delta \leq t \leq 0$. This implies that $\left|\psi_{2}(\kappa)\right| \leq \frac{M_{1}^{\prime}}{\kappa+\omega}$ for $\kappa \geq 0$ and hence, by Lemma $4.3 \psi_{2}$ belongs to $H$. Using Fubini's theorem and Lemma 4.1(ii) 
we observe that

$$
\begin{aligned}
& \int_{[0, \infty)}\left|0-\kappa \psi_{2}(\kappa)\right|^{2}(\kappa+\beta) \nu(d \kappa) \\
& \quad=\int_{[0, \infty)}\left|\psi_{2}(\kappa)\right|^{2}\left(\kappa^{3}+\beta \kappa^{2}\right) \nu(d \kappa) \\
& \quad \leq \int_{[0, \infty)}\left(\int_{0}^{\infty} e^{-\kappa s}\left|u_{2}(-s)\right| d s\right)\left(\int_{0}^{\infty} e^{-\kappa t}\left|u_{2}(-t)\right| d t\right)\left(\kappa^{3}+\beta \kappa^{2}\right) \nu(d \kappa) \\
& \quad=\int_{0}^{\infty} \int_{0}^{\infty}\left|u_{2}(-s)\right|\left|u_{2}(-t)\right|\left(-\frac{d^{3}}{d t^{3}} a(s+t)+\beta \frac{d^{2}}{d t^{2}} a(s+t)\right) d s d t .
\end{aligned}
$$

We split the last integral into four parts:

$$
\int_{0}^{\infty} \int_{0}^{\infty}=\int_{0}^{\delta} \int_{0}^{\delta}+\int_{\delta}^{\infty} \int_{\delta}^{\infty}+\int_{0}^{\delta} \int_{\delta}^{\infty}+\int_{\delta}^{\infty} \int_{0}^{\delta}
$$

We show that each integral at the righthand side is finite using Fubini's theorem, Lemma 4(i), (iv), (v), and (ix), and Hypothesis 3.5 (ii) and (iii). We start with the first integral:

$$
\begin{gathered}
\int_{0}^{\delta} \int_{0}^{\delta}\left|u_{2}(-s)\right|\left|u_{2}(-t)\right|\left(-\frac{d^{3}}{d t^{3}} a(s+t)+\beta \frac{d^{2}}{d t^{2}} a(s+t)\right) d s d t \\
\leq\left(M_{2}^{\prime}\right)^{2} \int_{s=0}^{\delta} s\left(\int_{t=0}^{\delta} t\left(-\frac{d^{3}}{d t^{3}} a(s+t)+\beta \frac{d^{2}}{d t^{2}} a(s+t)\right) d t\right) d s \\
=\left(M_{2}^{\prime}\right)^{2} \int_{s=0}^{\delta} s\left(\delta(\underbrace{\frac{d^{2}}{d t^{2}} a(s+\delta)+\beta \frac{d}{d t} a(s+\delta)}_{\leq 0})\right. \\
\quad \underbrace{\frac{d}{d t} a(s+\delta)-\beta a(s+\delta)}_{\leq 0}-\frac{d}{d t} a(s)+\beta a(s)) d s \\
\leq\left(M_{2}^{\prime}\right)^{2}\left(-\delta a(\delta)+\int_{0}^{\delta} a(s) d s+\beta \delta \int_{0}^{\delta} a(s) d s\right)<\infty .
\end{gathered}
$$


The second integral is finite, since

$$
\begin{aligned}
\int_{\delta}^{\infty} & \int_{\delta}^{\infty}\left|u_{2}(-s)\right|\left|u_{2}(-t)\right|\left(-\frac{d^{3}}{d t^{3}} a(s+t)+\beta \frac{d^{2}}{d t^{2}} a(s+t)\right) d s d t \\
& \leq\left(M_{1}^{\prime}\right)^{2} \int_{s=\delta}^{\infty}\left(\int_{t=\delta}^{\infty}\left(-\frac{d^{3}}{d t^{3}} a(s+t)+\beta \frac{d^{2}}{d t^{2}} a(s+t)\right) d t\right) d s \\
& =\left(M_{1}^{\prime}\right)^{2}\left(-\beta a(+\infty)-\frac{d}{d t} a(2 \delta)+\beta a(2 \delta)\right)<\infty
\end{aligned}
$$

Finiteness of the third integral, and analogously of the fourth, follows from

$$
\begin{aligned}
\int_{s=0}^{\delta} & \int_{t=\delta}^{\infty}\left|u_{2}(-s)\right|\left|u_{2}(-t)\right|\left(-\frac{d^{3}}{d t^{3}} a(s+t)+\beta \frac{d^{2}}{d t^{2}} a(s+t)\right) d s d t \\
& \leq M_{1}^{\prime} M_{2}^{\prime} \int_{s=0}^{\delta} s\left(\int_{t=\delta}^{\infty}\left(-\frac{d^{3}}{d t^{3}} a(s+t)+\beta \frac{d^{2}}{d t^{2}} a(s+t)\right) d t\right) d s \\
& =M_{1}^{\prime} M_{2}^{\prime}\left(\delta(\underbrace{\frac{d}{d t} a(2 \delta)-\beta a(2 \delta)}_{\leq 0})-a(2 \delta)+a(\delta)+\beta \delta a(\delta)\right)<\infty
\end{aligned}
$$

It follows that $\psi_{0}$ belongs to $D(J)$ and $J\left(\psi_{0}\right)=J\left(\psi_{1}\right)+J\left(\psi_{2}\right)=u_{0}(0)$. Finally, we show that $\psi_{0}$ belongs to $D(A)$, using the fact that $\nu(\{0\})=$ $a(+\infty)$, Fubini's theorem justified by Remark 3.6, Lemma 4.1(ii), and Hypothesis 3.5(iv):

$$
\begin{aligned}
\int_{[0, \infty)}\left(J\left(\psi_{0}\right)-\kappa \psi_{0}(\kappa)\right) \nu(d \kappa) \\
\quad=\int_{[0, \infty)}\left(1 \cdot u_{0}(0)-\kappa \int_{0}^{\infty} e^{-\kappa s} u_{0}(-s) d s\right) \nu(d \kappa) \\
=u_{0}(0) a(+\infty)+\int_{(0, \infty)}\left(\int_{0}^{\infty} \kappa e^{-\kappa s}\left(u_{0}(0)-u_{0}(-s)\right) d s\right) \nu(d \kappa) \\
=u_{0}(0) a(+\infty)+\int_{0}^{\infty}\left(-\frac{d}{d s} a(s)\right)\left(u_{0}(0)-u_{0}(-s)\right) d s=0
\end{aligned}
$$

$\square$

From semigroup theory we have the next proposition. 
Proposition 5.2. Problem (19) admits a unique strict solution $\psi$ : $[0, \infty) \rightarrow H$ in the sense that $\psi$ is continuous on $[0, \infty)$ with respect to the graph norm of $A$, continuously differentiable on $[0, \infty)$ with respect to the norm of $H$, and satisfies (19). Moreover, $\psi$ is real analytic on $(0, \infty)$ with respect to the graph norm of $A$ and $\psi(t)=S(t) \psi_{0}$ for $t \geq 0$.

Finally we prove Theorem 3.9.

Proof. From Proposition 5.2 and Lemmas 4.8 and 5.1 it is clear that $u$ given by $(10)$ is continuous on $[0, \infty)$ and real analytic on $(0, \infty)$, hence also Borel measurable. Using Hypothesis 3.5(ii), the continuity of $u$, and Lemma 4.1(i), we observe that for $t>0$

$$
\begin{aligned}
& \int_{-\infty}^{t} a(t-s)|u(s)| d s=\int_{-\infty}^{0} a(t-s)\left|u_{0}(s)\right| d s+\int_{0}^{t} a(t-s)|u(s)| d s \\
& \quad \leq M_{1} \int_{-\infty}^{t} e^{\omega s} a(t-s) d s+\max \{|u(s)| ; 0 \leq s \leq t\} \int_{0}^{t} a(t-s) d s \\
& \quad \leq M_{1} e^{\omega t} \hat{a}(\omega)+\max \{|u(s)| ; 0 \leq s \leq t\} \int_{0}^{t} a(s) d s<\infty .
\end{aligned}
$$

This implies that $u$ satisfies Definition 3.2(ii). To show that $u$ satisfies Definition 3.2(iii) and (iv) we observe that for $t \geq 0$

$$
\int_{-\infty}^{t} a(t-s) u(s) d s=\int_{-\infty}^{t}\left(\int_{[0, \infty)} e^{-\kappa(t-s)} \nu(d \kappa)\right) u(s) d s .
$$

Definition 3.2(ii) implies that we can apply Fubini's theorem to the righthand side of (20) to obtain for $t \geq 0$

$$
\begin{aligned}
\int_{-\infty}^{t}\left(\int_{[0, \infty)} e^{-\kappa(t-s)}\right. & \nu(d \kappa)) u(s) d s \\
& =\int_{[0, \infty)}\left(\int_{-\infty}^{t} e^{-\kappa(t-s)} u(s) d s\right) \nu(d \kappa) .
\end{aligned}
$$

We define $\psi(t):=S(t) \psi_{0}$ for $t \geq 0$ where $\psi_{0}$ is given by (3). Since $\psi(t)$ is an element of $H,(\psi(t))(\kappa)$ is defined only up to equality almost everywhere, for every $t \geq 0$. For further computation we need a Borel 
set $N \subseteq[0, \infty)$ of measure zero and a version $\underline{\psi}(t, \kappa)$ of $(\psi(t))(\kappa)$ that is differentiable and satisfies

$$
\begin{aligned}
\frac{\partial}{\partial t} \underline{\psi}(t, \kappa) & =J(\psi(t))-\kappa \underline{\psi}(t, \kappa), \quad t>0, \kappa \in[0, \infty) \backslash N, \\
\underline{\psi}(0, \kappa) & =\psi_{0}(\kappa), \quad \kappa \in[0, \infty) \backslash N .
\end{aligned}
$$

Since the semigroup $\{S(t)\}_{t \geq 0}$ is analytic, it can be proved, see $[\mathbf{1 5}, \mathrm{p}$. $72]$ and [7], that there exist a function $\psi:[0, \infty) \times[0, \infty) \rightarrow \mathbf{R}$ and a Borel set $N \subseteq[0, \infty)$ of measure zero such that for $\kappa \in[0, \infty) \backslash N$ the function $t \mapsto \psi(t, \kappa)$ is continuous on $[0, \infty)$, analytic on $(0, \infty)$, and $\underline{\psi}(t, \kappa)=(\psi(t) \overline{)}(\kappa)$ for $t \geq 0$. Moreover,(22) holds and hence, we have using (10)

$$
\begin{aligned}
\underline{\psi}(t, \kappa) & =e^{-\kappa t} \psi_{0}(\kappa)+\int_{0}^{t} e^{-\kappa(t-s)} J(\psi(s)) d s \\
& =\int_{-\infty}^{t} e^{-\kappa(t-s)} u(s) d s, \quad t \geq 0
\end{aligned}
$$

as well as

$$
\underline{\psi}(t, \kappa)=\psi_{0}(\kappa)+\int_{0}^{t}(J(\psi(s))-\kappa \underline{\psi}(s, \kappa)) d s, \quad t \geq 0 .
$$

Therefore we have for $t \geq 0$

$$
\begin{aligned}
\int_{[0, \infty)} & \left(\int_{-\infty}^{t} e^{-\kappa(t-s)} u(s) d s\right) \nu(d \kappa)=\int_{[0, \infty)} \underline{\psi}(t, \kappa) \nu(d \kappa) \\
= & \int_{[0, \infty)} \psi_{0}(\kappa) \nu(d \kappa)+\int_{[0, \infty)}\left(\int_{0}^{t}(J(\psi(s))-\kappa \underline{\psi}(s, \kappa)) d s\right) \nu(d \kappa) .
\end{aligned}
$$

To show that we can apply Fubini's theorem in (23) it is sufficient to show that the function $s \mapsto \int_{[0, \infty)}(J(\psi(s))-\kappa \underline{\psi}(s, \kappa)) \nu(d \kappa)$ is bounded on $[0, t]$ for every $t \geq 0$. Using Lemma 4.2 and the fact that $\psi$ is continuous on $[0, \infty)$ with respect to the graph norm of $A$, denoted by $\|\cdot\|_{A}$, this follows from

$$
\begin{aligned}
& \int_{[0, \infty)}|J(\psi(s))-\kappa \underline{\psi}(s, \kappa)| \nu(d \kappa)=\int_{[0, \infty)}|(A \psi(s))(\kappa)| \nu(d \kappa) \\
& \leq \sqrt{\hat{a}(\beta)}\|\psi(s)\|_{A} \leq \sqrt{\hat{a}(\beta)} \quad \max \left\{\|\psi(s)\|_{A} ; 0 \leq s \leq t\right\} .
\end{aligned}
$$


Thus we have for $t \geq 0$

$$
\begin{aligned}
& \int_{[0, \infty)} \psi_{0}(\kappa) \nu(d \kappa)+\int_{[0, \infty)}\left(\int_{0}^{t}(J(\psi(s))-\kappa \underline{\psi}(s, \kappa)) d s\right) \nu(d \kappa) \\
& =\int_{[0, \infty)} \psi_{0}(\kappa) \nu(d \kappa)+\int_{0}^{t}\left(\int_{[0, \infty)}(J(\psi(s))-\kappa(\psi(s))(\kappa)) \nu(d \kappa)\right) d s .
\end{aligned}
$$

Combining (20), (21), (23), and (24), and recalling that $\psi(s)$ belongs to $D(A)$ for $s \geq 0$ it follows that

$$
\int_{-\infty}^{t} a(t-s) u(s) d s=\int_{[0, \infty)} \psi_{0}(\kappa) \nu(d \kappa), \quad t \geq 0 .
$$

This shows that $u$ satisfies Definition 3.2(iii) and (iv), since the function $v$ is constant.

Extrapolation and interpolation spaces. The main results of this section are Propositions 6.5 and 6.6. Before we are in a position to state the former we need some preliminaries.

The extrapolation space $H_{-1}$ generated by $A$ is defined as the completion of $H$ with respect to the norm $\|\cdot\|_{-1}$ defined by $\|\varphi\|_{-1}:=\|(\beta I-$ $A)^{-1} \varphi \|$ for $\varphi \in H$. The semigroup $\{S(t)\}_{t \geq 0}$ extends continuously to a strongly continuous, positive, analytic semigroup $\left\{S_{-1}(t)\right\}_{t \geq 0}$ on $H_{-1}$. Moreover, there exists a unique continuous extension $A_{-1}: D\left(A_{-1}\right)=$ $H \subseteq H_{-1} \rightarrow H_{-1}$ of $A$ such that $A_{-1}$ is the infinitesimal generator of $\left\{S_{-1}(t)\right\}_{t \geq 0}$. See [2, pp. 18-20] and [1, p. 262].

The following lemma concerns the dual space $\left(H_{-1}\right)^{\prime}$ of the extrapolation space $H_{-1}$. For a proof we refer to [1, p. 270].

Lemma 6.1. $\left(H_{-1}\right)^{\prime}=D\left(A^{*}\right)$ and the corresponding norms are equivalent.

Now we introduce interpolation spaces, which are useful tools to handle questions of regularity. See $[\mathbf{1}, \mathbf{1 0}]$. The real interpolation space $(H, D(A))_{\alpha, 2}$ with $0<\alpha<1$ is denoted by $D_{A}(\alpha, 2)$, see $[\mathbf{1 0}$, p. 46]. 
The next corollary concerns the dual space $\left(H_{-1}, H\right)_{\eta, 2}^{\prime}$ of the interpolation space $\left(H_{-1}, H\right)_{\eta, 2}$ with $0<\eta<1$. For a proof we refer to $[\mathbf{1 6}$, pp. 25, 69] and Lemma 6.1.

Corollary 6.2. If $0<\eta<1$, then

$$
\left(H_{-1}, H\right)_{\eta, 2}^{\prime}=D_{A^{*}}(1-\eta, 2)=D_{\beta I-A^{*}}(1-\eta, 2)
$$

and the corresponding norms are equivalent.

Definition 6.3. The function $\xi:[0, \infty) \rightarrow \mathbf{R}$ is defined by

$$
\xi(\kappa):=\frac{\kappa}{(\kappa+\beta)^{2}}, \quad \kappa \in[0, \infty) .
$$

Note that $\xi$ belongs to $H$ as a consequence of Lemma 4.3.

Proposition 6.4. If $0<\eta<1$, then $\xi$ belongs to $D_{\beta I-A^{*}}(1-\eta, 2)$ if and only if Hypothesis 3.10(i) holds.

Proof. Let $0<\eta<1$. By definition of the interpolation space $D_{\beta I-A^{*}}(1-\eta, 2)$, see $[\mathbf{1 0}$, p. 47$], \xi$ belongs to $D_{\beta I-A^{*}}(1-\eta, 2)$ if

$$
\int_{c}^{\infty} t^{2(1-\eta)-1}\left\|\left(\beta I-A^{*}\right)\left(t I-\left(\beta I-A^{*}\right)\right)^{-1} \xi\right\|^{2} d t<\infty
$$

for $c>0$ sufficiently large. This holds if and only if

$$
\int_{c}^{\infty} t^{1-2 \eta}\left\|\left(\beta I-A^{*}\right)\left(t I-A^{*}\right)^{-1} \xi\right\|^{2} d t<\infty .
$$

Using Proposition 4.10 with $p \in \mathbf{C}$ such that

$$
\begin{aligned}
p & =\frac{1}{t \hat{a}(t)} \int_{[0, \infty)} \frac{\kappa+\beta}{\kappa+t} \xi(\kappa) \nu(d \kappa) \\
& =\frac{1}{t \hat{a}(t)} \int_{[0, \infty)} \frac{\kappa+\beta}{\kappa+t} \frac{\kappa}{(\kappa+\beta)^{2}} \nu(d \kappa) \\
& =\frac{1}{t \hat{a}(t)} \int_{[0, \infty)} \frac{1}{t-\beta}\left(\frac{t}{\kappa+t}-\frac{\beta}{\kappa+\beta}\right) \nu(d \kappa) \\
& =\frac{1}{t-\beta}\left(1-\frac{\beta \hat{a}(\beta)}{t \hat{a}(t)}\right),
\end{aligned}
$$


it follows by straightforward calculation that for $t>0$

$$
\begin{aligned}
\left(\beta I-A^{*}\right)\left(t I-A^{*}\right)^{-1} \xi & =\xi-(t-\beta)\left(t I-A^{*}\right)^{-1} \xi \\
& =\xi-(t-\beta) \frac{1}{\underline{\kappa}+t}\left(\xi+p \frac{\underline{\kappa}}{\underline{\kappa}+\beta}\right) \\
& =\frac{\beta \hat{a}(\beta)}{t \hat{a}(t)} \frac{\underline{\kappa}}{(\underline{\kappa}+\beta)(\underline{\kappa}+t)} .
\end{aligned}
$$

Hence, using Lemma 4.1(xiii) we have that for $t>0$

$$
\begin{aligned}
& \left\|\left(\beta I-A^{*}\right)\left(t I-A^{*}\right)^{-1} \xi\right\|^{2} \\
& \begin{aligned}
=\beta^{2}(\hat{a}(\beta))^{2}\left(\frac{\hat{a}(t)+t(d / d) \hat{a}(t)}{t^{2}(\hat{a}(t))^{2}}\right. & +\beta \frac{(d / d) \hat{a}(t)}{t(t-\beta)(\hat{a}(t))^{2}} \\
& \left.+\beta^{2} \frac{\hat{a}(\beta)-\hat{a}(t)}{t^{2}(\hat{a}(t))^{2}(t-\beta)^{2}}\right) .
\end{aligned}
\end{aligned}
$$

By Lemma 4.1(xvi), (xv), and (xiv), and by the fact that

$$
\frac{1}{t} \leq \frac{1}{t-\beta} \leq \frac{c}{c-\beta} \frac{1}{t}
$$

for $t>c$ whenever $c>\beta$, we observe that for $c>\beta$

$$
\int_{c}^{\infty} t^{1-2 \eta} \frac{\hat{a}(\beta)-\hat{a}(t)}{t^{2}(\hat{a}(t))^{2}(t-\beta)^{2}} d t \leq \frac{1}{\beta} \frac{1}{c-\beta} \frac{1}{\hat{a}(c)} \int_{c}^{\infty} t^{-1-2 \eta} d t<\infty
$$

and

$$
\int_{c}^{\infty} t^{1-2 \eta} \frac{-(d / d t) \hat{a}(t)}{t(t-\beta)(\hat{a}(t))^{2}} d t \leq \frac{1}{c-\beta} \frac{1}{\hat{a}(c)} \int_{c}^{\infty} t^{-1-2 \eta} d t<\infty .
$$

Combined with (26) this implies that (25) holds if and only if

$$
\int_{c}^{\infty} t^{-1-2 \eta} \frac{\hat{a}(t)+t(d / d t) \hat{a}(t)}{(\hat{a}(t))^{2}} d t<\infty
$$

with $c>\beta$, which is equivalent to Hypothesis 3.10(i).

Proposition 6.5. Let $0<\eta<1$. The restriction $\left.J\right|_{D(A)}$ may be extended continuously to a linear bounded functional from $D_{A}(\eta, 2)$ into $\mathbf{C}$ if and only if Hypothesis 3.10(i) holds. 
Proof. By Proposition 6.4 it is sufficient to prove that $\left.J\right|_{D(A)}$ may be extended continuously to a linear bounded functional from $D_{A}(\eta, 2)$ into $\mathbf{C}$ if and only if $\xi$ belongs to $D_{\beta I-A^{*}}(1-\eta, 2)$. We shall use the fact that $\left.J\right|_{D_{A}}=J \circ(\beta I-A)^{-1} \circ(\beta I-A)$. We first prove the 'if' part of the proposition. Let us assume that $\xi$ belongs to $D_{\beta I-A^{*}}(1-\eta, 2)$. Corollary 6.2 implies that there exists a linear bounded functional $T \in\left(H_{-1}, H\right)_{\eta, 2}^{\prime}$ such that $T(\varphi)=\langle\varphi, \xi\rangle$ for $\varphi \in H$. Using Proposition 4.7 we therefore have for $\varphi \in H$

$$
\begin{aligned}
\left|\left(J \circ(\beta I-A)^{-1}\right)(\varphi)\right| & =\left|\frac{1}{\beta \hat{a}(\beta)} \int_{[0, \infty)} \frac{\kappa \varphi(\kappa)}{\kappa+\beta} \nu(d \kappa)\right| \\
& =\frac{1}{\beta \hat{a}(\beta)}|\langle\varphi, \xi\rangle|=\frac{1}{\beta \hat{a}(\beta)}|T(\varphi)| \\
& \leq \frac{1}{\beta \hat{a}(\beta)}\|T\|_{\left(H_{-1}, H\right)_{\eta, 2}^{\prime}}\|\varphi\|_{\left(H_{-1}, H\right)_{\eta, 2}} .
\end{aligned}
$$

Hence, $J \circ(\beta I-A)^{-1}:\left(H,\|\cdot\|_{\left(H_{-1}, H\right)_{\eta, 2}}\right) \rightarrow \mathbf{C}$ is a linear bounded functional. As $H$ is dense in $\left(H_{-1}, H\right)_{\eta, 2}$, see [10, p. 23], there exists a continuous extension of $J \circ(\beta I-A)^{-1}$ that we denote by $E:\left(H_{-1}, H\right)_{\eta, 2} \rightarrow \mathbf{C}$. The linear operator $\beta I-A: D(A) \rightarrow H$ is bounded, where $D(A)$ is equipped with the graph norm of $A$. Moreover, the linear operator $\beta I-A_{-1}: H \rightarrow H_{-1}$ is bounded, see [1, pp. 262, 2]. Furthermore, we have the continuous embeddings $D(A) \hookrightarrow H \hookrightarrow H_{-1}$, see [1, pp. 256, 262]. It is a consequence of interpolation theory that $\beta I-A_{-1}: D_{A}(\eta, 2) \rightarrow\left(H_{-1}, H\right)_{\eta, 2}$ is a linear bounded operator, see [10, p. 19]. Therefore $E \circ\left(\beta I-A_{-1}\right): D_{A}(\eta, 2) \rightarrow \mathbf{C}$ is a linear bounded functional and for $\psi \in D(A)$ we have

$$
\begin{aligned}
\left(E \circ\left(\beta I-A_{-1}\right)\right)(\psi) & =E((\beta I-A) \psi) \\
& =\left(J \circ(\beta I-A)^{-1}\right)((\beta I-A) \psi)=J(\psi) .
\end{aligned}
$$

This shows that $\left.E \circ\left(\beta I-A_{-1}\right)\right|_{D_{A}(\eta, 2)}$ is a continuous extension of $\left.J\right|_{D(A)}$.

We now prove the 'only if' part of the proposition. Let us assume that $\left.J\right|_{D(A)}$ extends continuously to a linear bounded functional from $D_{A}(\eta, 2)$ into C. For our convenience we denote this extension by $J: D_{A}(\eta, 2) \rightarrow \mathbf{C}$. Since the linear operators $(\beta I-A)^{-1}: H \rightarrow D(A)$ and $\left(\beta I-A_{-1}\right)^{-1}: H_{-1} \rightarrow H$ are bounded, see Proposition 4.7 
respectively [1, pp. 262, 2], and the embeddings $H \hookrightarrow\left(H_{-1}, H\right)_{\eta, 2} \hookrightarrow$ $H_{-1}$ are continuous, see $\left[\mathbf{1 0}\right.$, p. 16], we have that $\left(\beta I-A_{-1}\right)^{-1}$ : $\left(H_{-1}, H\right)_{\eta, 2} \rightarrow D_{A}(\eta, 2)$ is a linear bounded operator, see [10, p. 19]. Hence, $J \circ\left(\beta I-A_{-1}\right)^{-1}:\left(H_{-1}, H\right)_{\eta, 2} \rightarrow \mathbf{C}$ is a linear bounded functional. Using Proposition 4.7 we therefore have for $\varphi \in H$

$$
\begin{aligned}
|\langle\varphi, \xi\rangle| & =\left|\int_{[0, \infty)} \frac{\kappa \varphi(\kappa)}{\kappa+\beta} \nu(d \kappa)\right| \\
& =\beta \hat{a}(\beta)\left|\left(J \circ\left(\beta I-A_{-1}\right)^{-1}\right)(\varphi)\right| \\
& \leq \beta \hat{a}(\beta)\left\|J \circ\left(\beta I-A_{-1}\right)^{-1}\right\|_{\left(H_{-1}, H\right)_{\eta, 2}^{\prime}}\|\varphi\|_{\left(H_{-1}, H\right)_{\eta, 2}} .
\end{aligned}
$$

This shows that $\varphi \mapsto\langle\varphi, \xi\rangle$ is a linear bounded functional on $\left(H,\|\cdot\|_{\left(H_{-1}, H\right)_{\eta, 2}}\right)$. As $H$ is dense in $\left(H_{-1}, H\right)_{\eta, 2}$, this functional extends continuously to a linear bounded functional $T \in\left(H_{-1}, H\right)_{\eta, 2}^{\prime}$ that satisfies $T(\varphi)=\langle\varphi, \xi\rangle$ for $\varphi \in H$. It is a result of Corollary 6.2 that $\xi$ belongs to $D_{\beta I-A^{*}}(1-\eta, 2)$.

Proposition 6.6. Let $0<\theta<1$. The function $\pi \in H$ given by (11) belongs to $D_{A}(\theta, 2)$ if and only if Hypothesis 3.10(ii) holds.

Proof. It is sufficient to prove Remark 3.13 for the function $\tilde{\pi}:=(1 / \underline{\kappa}+\beta)$. Let $0<\theta<1$. By definition of the interpolation space $D_{A}(\theta, 2)$, see $\left[\mathbf{1 0}\right.$, p. 49], $\tilde{\pi}$ belongs to $D_{A}(\theta, 2)$ if

$$
\int_{c}^{\infty} t^{2 \theta-1}\left\|A(t I-A)^{-1} \tilde{\pi}\right\|^{2} d t<\infty
$$

for $c>0$ sufficiently large. Using Proposition 4.7 with $u \in \mathbf{C}$ such that

$$
\begin{aligned}
u & =\frac{1}{t \hat{a}(t)} \int_{[0, \infty)} \frac{\kappa \tilde{\pi}(\kappa)}{\kappa+t} \nu(d \kappa) \\
& =\frac{1}{t \hat{a}(t)} \int_{[0, \infty)} \frac{\kappa}{\kappa+t} \frac{1}{\kappa+\beta} \nu(d \kappa) \\
& =\frac{1}{t \hat{a}(t)} \int_{[0, \infty)} \frac{1}{t-\beta}\left(\frac{t}{\kappa+t}-\frac{\beta}{\kappa+\beta}\right) \nu(d \kappa) \\
& =\frac{1}{t(t-\beta) \hat{a}(t)}(t \hat{a}(t)-\beta \hat{a}(\beta)),
\end{aligned}
$$


we have for $t>0$

$$
\begin{aligned}
A(t I-A)^{-1} \tilde{\pi} & =-\tilde{\pi}+t(t I-A)^{-1} \tilde{\pi}=-\tilde{\pi}+t \frac{\tilde{\pi}(\underline{\kappa})+u}{\underline{\kappa}+t} \\
& =u t \frac{1}{\underline{\kappa}+t}-\frac{\underline{\kappa}}{\underline{\kappa}+t} \tilde{\pi}(\underline{\kappa}) .
\end{aligned}
$$

Hence, by straightforward calculation using 4.1(xii) and (xiii) we have for $t>0$

$$
\begin{aligned}
& \left\|A(t I-A)^{-1} \tilde{\pi}\right\|^{2} \\
& =\beta^{2}(\hat{a}(\beta))^{2}\left(-\frac{1}{\hat{a}(\beta)} \frac{1}{(t-\beta)^{2}}+\frac{\hat{a}(t)+t(d / d t) \hat{a}(t)}{(t-\beta)^{2}(\hat{a}(t))^{2}}-\beta \frac{(d / d t) \hat{a}(t)}{(t-\beta)^{2}(\hat{a}(t))^{2}}\right) .
\end{aligned}
$$

By (27) and Lemma 4.1(xiv) and (xv) we observe that for $c>\beta$

$$
\int_{c}^{\infty} t^{2 \theta-1} \frac{1}{(t-\beta)^{2}} d t \leq\left(\frac{c}{c-\beta}\right)^{2} \int_{c}^{\infty} t^{2 \theta-3} d t<\infty
$$

and

$$
\begin{aligned}
\int_{c}^{\infty} t^{2 \theta-1} \frac{-(d / d t) \hat{a}(t)}{(t-\beta)^{2}(\hat{a}(t))^{2}} d t & \leq\left(\frac{c}{c-\beta}\right)^{2} \int_{c}^{\infty} t^{2 \theta-3} \frac{1}{t \hat{a}(t)} d t \\
& \leq\left(\frac{c}{c-\beta}\right)^{2} \frac{1}{c \hat{a}(c)} \int_{c}^{\infty} t^{2 \theta-3} d t<\infty .
\end{aligned}
$$

Combined with (29) this implies that (28) holds if and only if

$$
\int_{c}^{\infty} t^{2 \theta-1} \frac{\hat{a}(t)+t(d / d t) \hat{a}(t)}{(t-\beta)^{2}(\hat{a}(t))^{2}} d t<\infty
$$

with $c>\beta$, which is equivalent to Hypothesis 3.10(ii). $\quad \square$

Note that Propositions 6.5 and 6.6 imply Remarks 3.12 and 3.13, respectively. It is easily observed that Remark 3.11 follows by using Lemma 4.1(xiv).

7. The homogeneous case II. With the results of Section 6 we are now in a position to prove Theorem 3.15. 
Proof. Let the functions $u_{1}, u_{2}:(-\infty, 0] \rightarrow \mathbf{R}$ and $\psi_{1}, \psi_{2}:[0, \infty) \rightarrow$ $\mathbf{R}$ be defined by respectively $u_{1}(t):=u_{0}(t)-\gamma e^{\beta t}$ and $u_{2}(t):=\gamma e^{\beta t}$ for $t \leq 0$ and

$$
\psi_{j}(\kappa):=\int_{0}^{\infty} e^{-\kappa s} u_{j}(-s) d s, \quad \kappa \geq 0, j=1,2
$$

where

$$
\gamma:=\frac{u_{0}(0) a(+\infty)}{\beta \hat{a}(\beta)}+\frac{1}{\beta \hat{a}(\beta)} \int_{0}^{\infty}\left(-\frac{d}{d t} a(t)\right)\left(u_{0}(0)-u_{0}(-t)\right) d t .
$$

Note that $u_{0}=u_{1}+u_{2}$ and $\psi_{0}=\psi_{1}+\psi_{2}$. It is easily seen that $u_{1}$ and $u_{2}$ satisfy Hypothesis 3.5(i), (ii) and (iii). Furthermore, it follows from straightforward calculation using the definition of $\gamma$ and Lemma 4.1(iv) and (ix) that $u_{1}$ also satisfies Hypothesis 3.5(iv). Therefore Theorem 3.9 implies that the function $\tilde{u}_{1}: \mathbf{R} \rightarrow \mathbf{R}$ defined by

$$
\tilde{u}_{1}(t):= \begin{cases}J\left(S(t) \psi_{1}\right) & t>0 \\ u_{1}(t) & t \leq 0\end{cases}
$$

is the unique solution to (1) with $f$ identically zero and $u_{0}$ replaced by $u_{1}$, and $\tilde{u}_{1}$ is continuous on $[0, \infty)$ and real analytic on $(0, \infty)$. Since $\psi_{1}$ belongs to $D(A)$, which can be proved in the same way as Lemma 5.1. Proposition A.2(ii) implies that $t \mapsto S(t) \psi_{1}$ belongs to $C^{1-\eta}\left([0, \infty) ; D_{A}(\eta, 2)\right)$. As $J: D_{A}(\eta, 2) \rightarrow \mathbf{C}$ is continuous by Remark 3.12 , this implies that $\tilde{u}_{1}$ belongs to $C^{1-\eta}[0, \infty)$.

Recalling that the function $b:(0, \infty) \rightarrow \mathbf{R}$ is the integral resolvent of the first kind of $a$, see (2), and extending $u_{2}$ to $u_{2}: \mathbf{R} \rightarrow \mathbf{R}$ by $u_{2}(t):=\gamma e^{\beta t}$ for $t \in \mathbf{R}$, it follows from Proposition B.1 that the function $\tilde{u}_{2}: \mathbf{R} \rightarrow \mathbf{R}$ defined by

$$
\tilde{u}_{2}(t):= \begin{cases}u_{2}(t)-\beta \hat{a}(\beta) \int_{0}^{t} b(t-s) u_{2}(s) d s & t>0, \\ u_{2}(t) & t \leq 0,\end{cases}
$$

is the unique solution to (1) with $f$ identically zero and $u_{0}$ replaced by $u_{2}$. We shall prove that $\tilde{u}_{2}(t)=J\left(S(t) \psi_{2}\right)$ for $t>0$. Note that the righthand side is well-defined, since

$$
\psi_{2}(\kappa)=\gamma \int_{0}^{\infty} e^{-\kappa s} e^{-\beta s} d s=\gamma \frac{1}{\kappa+\beta}=\beta \hat{a}(\beta) \gamma \pi(\kappa), \quad \kappa \geq 0,
$$


where $\pi \in H$ is given by (11). By semigroup theory we have $(s I-A)^{-1}=\int_{0}^{\infty} e^{-s t} S(t) d t$ for $s>\beta / 4$, see [10, p. 40]. Moreover, $\int_{0}^{\infty} e^{-s t} S(t) \pi d t$ exists as a Bochner integral in $D_{A}(\eta, 2)$ for $s>\beta / 4$, since $\pi$ belongs to $D_{A}(\theta, 2)$ by Remark 3.13 and $\|S(t)\|_{\mathcal{L}\left(D_{A}(\eta, 2)\right)} \leq$ $e^{(\beta / 4) t}$ for $t>0$ by interpolation theory. Using the continuity of $J: D_{A}(\eta, 2) \rightarrow \mathbf{C}$, Proposition 4.7, and the fact that the Laplace transform of a convolution is the product of the individual Laplace transforms, in particular $\hat{a}(s) \hat{b}(s)=1 / s$ and $\hat{u}_{2}(s)=\gamma \frac{1}{s-\beta}$ for $s>0$, we have for $s>\beta$

$$
\begin{aligned}
\int_{0}^{\infty} e^{-s t} J\left(S(t) \psi_{2}\right) d t \\
=\beta \hat{a}(\beta) \gamma \int_{0}^{\infty} e^{-s t} J(S(t) \pi) d t \\
=\beta \hat{a}(\beta) \gamma J\left((s I-A)^{-1} \pi\right) \\
=\beta \hat{a}(\beta) \gamma \frac{1}{s \hat{a}(s)} \int_{[0, \infty)} \frac{\kappa \pi(\kappa)}{\kappa+s} \nu(d \kappa) \\
=\gamma \frac{1}{s \hat{a}(s)} \int_{[0, \infty)} \frac{\kappa}{(\kappa+s)(\kappa+\beta)} \nu(d \kappa) \\
=\gamma \frac{1}{s \hat{a}(s)} \frac{1}{s-\beta}(s \hat{a}(s)-\beta \hat{a}(\beta)) \\
=\hat{u}_{2}(s)(1-\beta \hat{a}(\beta) \hat{b}(s)) \\
=\int_{0}^{\infty} e^{-s t}\left(u_{2}(t)-\beta \hat{a}(\beta) \int_{0}^{t} b(t-\sigma) u_{2}(\sigma) d \sigma\right) d t \\
=\int_{0}^{\infty} e^{-s t} \tilde{u}_{2}(t) d t .
\end{aligned}
$$

This shows that the Laplace transforms of $\tilde{u}_{2}$ and $t \mapsto J\left(S(t) \psi_{2}\right)$ are equal on $(\beta, \infty)$. It is a consequence of the inversion theorem for Laplace transforms that $\tilde{u}_{2}(t)=J\left(S(t) \psi_{2}\right)$ for $t>0$. As $\pi$, and thus $\psi_{2}$, belongs to $D_{A}(\theta, 2)$ with $D_{A}(\theta, 2)$ embedded in $D_{A}(\eta, 2)$, see [10, p. 16], and as $J: D_{A}(\eta, 2) \rightarrow \mathbf{C}$ is continuous, it is clear that $\tilde{u}_{2}$ is real analytic on $(0, \infty)$. Moreover, $\tilde{u}_{2}$ belongs to $C^{\theta-\eta}[0, \infty)$ as a consequence of Proposition A.2(iii).

It follows that the function $u: \mathbf{R} \rightarrow \mathbf{R}$ defined by $u:=\tilde{u}_{2}+\tilde{u}_{2}$ is the unique solution to (1) with $f$ identically zero, and $u$ belongs to 
$C^{\theta-\eta}[0, \infty)$, is real analytic on $(0, \infty)$, and satisfies $(10)$.

Finally we prove the equality in Remark 3.16.

Proof. By semigroup theory we have $(s I-A)^{-1}=\int_{0}^{\infty} e^{-s t} S(t) d t$ for $s>(\beta / 4)$, see $[\mathbf{1 0}$, p. 40]. Using Proposition 4.7 and the continuity of $J: D_{A}(\eta, 2) \rightarrow \mathbf{C}$ by Remark 3.12, we have for $s>(\beta / 4)$ and $\kappa \geq 0$

$$
\int_{0}^{\infty} e^{-s t}\left(S(t) \psi_{0}\right)(\kappa) d t=\left((s I-A)^{-1} \psi_{0}\right)(\kappa)=\frac{\psi_{0}(\kappa)+p}{\kappa+s}
$$

where $p \in \mathbf{C}$ satisfies

$$
p=J\left((s I-A)^{-1} \psi_{0}\right)=\int_{0}^{\infty} e^{-s t} J\left(S(t) \psi_{0}\right) d t=\hat{u}(s) .
$$

As the Laplace transform of a convolution is the product of the individual Laplace transforms, it follows that for $s>\beta / 4$ and $\kappa \geq 0$

$$
\begin{aligned}
\int_{0}^{\infty} e^{-s t}\left(S(t) \psi_{0}\right)(\kappa) d t \\
\quad=\frac{\psi_{0}(\kappa)}{\kappa+s}+\frac{\hat{u}(s)}{\kappa+s} \\
\quad=\int_{0}^{\infty} e^{-s t}\left(e^{-\kappa t} \psi_{0}(\kappa)+\int_{0}^{t} e^{-\kappa(t-\sigma)} u(\sigma) d \sigma\right) d t \\
=\int_{0}^{\infty} e^{-s t}\left(e^{-\kappa t} \int_{0}^{\infty} e^{-\kappa \sigma} u_{0}(-\sigma) d \sigma+\int_{0}^{t} e^{-\kappa(t-\sigma)} u(\sigma) d \sigma\right) d t \\
=\int_{0}^{\infty} e^{-s t}\left(\int_{0}^{\infty} e^{-\kappa \sigma} u(t-\sigma) d \sigma\right) d t .
\end{aligned}
$$

The remark is a consequence of the inversion theorem for Laplace transforms.

8. The inhomogeneous case. The goal of this section is to prove Theorem 3.17. To this end we consider the inhomogeneous problem (1) with $u_{0}$ identically zero and $f$ locally integrable. Analogous to Section 5 we first want to consider (4), with $\psi_{0}$ identically zero, as an abstract Cauchy problem in an appropriate space. Therefore we 
define the linear operator $\tilde{A}: D(J) \rightarrow H$ by $\tilde{A} \psi:=J(\psi) \mathbf{1}-\underline{\kappa} \psi(\underline{\kappa})$ for $\psi \in D(J)$ and the linear functional $I: D(J) \rightarrow \mathbf{C}$ by

$$
I(\psi):=\int_{[0, \infty)}(J(\psi)-\kappa \psi(\kappa)) \nu(d \kappa), \quad \psi \in D(J) .
$$

Note that $\left.\tilde{A}\right|_{D(A)}=A$, that $I$ is well-defined by Lemma 4.2 , and that if $\psi$ belongs to $D(J)$, then $\psi$ belongs to $D(A)$ if and only if $I(\psi)=0$. Recalling that $\pi$ is given by (11) the next lemma is easily seen.

Lemma 8.1. The function $\pi$ belongs to $D(J), J(\pi)=\frac{1}{\beta \hat{a}(\beta)}$, $\tilde{A} \pi=\beta \pi$ and $I(\pi)=1$. Moreover, if $\psi \in D(J)$, then $\psi-I(\psi) \pi$ belongs to $D(A)$.

Heuristically the semigroup approach continues as follows. Since for $t>0, \psi(t, \cdot)$ satisfies (5) with $u(t)=J(\psi(t, \cdot))$, that is, $I(\psi(t, \cdot))=f(t)$ where $f$ is not identically zero, $\psi(t, \cdot)$ does not belong to $D(A)$. Using Lemma 8.1 we rewrite (4) into

$$
\begin{aligned}
\frac{d}{d t} \psi(t, \cdot) & =\tilde{A} \psi(t, \cdot)=A(\psi(t, \cdot)-f(t) \pi)+f(t) \tilde{A} \pi \\
& =A_{-1}(\psi(t, \cdot)-f(t) \pi)+\beta f(t) \pi \\
& =A_{-1} \psi(t, \cdot)+f(t)\left(\beta I-A_{-1}\right) \pi
\end{aligned}
$$

for $t>0$ and $\psi(0, \cdot)=0$. In view of the semigroup setting we therefore study the following inhomogeneous abstract Cauchy problem in $H_{-1}$ :

$$
\begin{aligned}
\frac{d}{d t} \psi(t) & =A_{-1} \psi(t)+f(t)\left(\beta I-A_{-1}\right) \pi, \quad t>0, \\
\psi(0) & =0 .
\end{aligned}
$$

From semigroup theory we have the next proposition.

Proposition 8.2. Problem (30) admits a unique mild solution $\psi$ : $[0, \infty) \rightarrow H_{-1}$ in the sense that $\psi(t)=\int_{0}^{t} S_{-1}(t-s) f(s)\left(\beta I-A_{-1}\right) \pi d s$ for $t>0$ where the integral is a Bochner integral in $H_{-1}$. Moreover, $\psi$ is continuous on $[0, \infty)$ with respect to the norm of $H_{-1}$ and $\psi(t)=$ 
$\left(\beta I-A_{-1}\right) \int_{0}^{t} S(t-s) f(s) \pi d s$ for $t>0$ where the integral is a Bochner integral in $H$.

The following proposition gives conditions under which the mild solution to (30) has values in $D_{A}(\eta, 2)$ with $0<\eta<1$.

Proposition 8.3. Let a satisfy Hypothesis 3.10 with $0<\eta<\theta<1$. Let $f$ belong to $L^{p}[0, T]$ for every $T>0$ where $1 \leq p<\infty$. Then the mild solution $\psi$ to (30) is given by $\psi(t)=\int_{0}^{t}(\beta I-A) S(t-s) f(s) \pi d s$ for $t>0$ where the integral is a Bochner integral in $D_{A}(\eta, 2)$. Moreover, the following holds:

(i) If $p=1$, then $\psi$ belongs to $L^{q}\left([0, T] ; D_{A}(\eta, 2)\right)$ for every

$$
1 \leq q<\frac{1}{1-(\theta-\eta)} \text { and } T>0
$$

(ii) If $1<p<\frac{1}{\theta-\eta}$, then $\psi$ belongs to $L^{q}\left([0, T] ; D_{A}(\eta, 2)\right)$ for every

$$
1 \leq q \leq \frac{p}{1-(\theta-\eta) p} \text { and } T>0
$$

(iii) If $p=\frac{1}{\theta-\eta}$, then $\psi$ belongs to $L^{q}\left([0, T] ; D_{A}(\eta, 2)\right)$ for every

$$
1 \leq q<\infty \text { and } T>0
$$

(iv) If $\frac{1}{\theta-\eta}<p<\infty$, then $\psi$ belongs to $C_{0}^{\theta-\eta-(1 / p)}\left([0, T] ; D_{A}(\eta, 2)\right)$

$$
\text { for every } T>0 \text {. }
$$

Proof. Properties (i)-(iv) follow by applying Proposition A.2(iv)-(vii). We only have to prove that $\int_{0}^{t}(\beta I-A) S(t-s) f(s) \pi d s$ exists as a Bochner integral in $D_{A}(\eta, 2)$ for $0<t \leq T$ whenever $\frac{1}{\theta-\eta}<p<\infty$. Note that $\pi$ belongs to $D_{A}(\theta, 2)$ by Remark 3.13 and that $D_{A}(\theta, 2)$ is embedded in $D_{A}(\eta, 2)$. Let $T>0$ and $\frac{1}{\theta-\eta}<p<\infty$. Let $1<p^{\prime}<\infty$ be such that $\frac{1}{p}+\frac{1}{p^{\prime}}=1$; hence, $(-1+\theta-\eta) p^{\prime}>-1$. Combining semigroup and interpolation theory it follows that there exists $C>0$ such that $\|(\beta I-A) S(t)\|_{\mathcal{L}\left(D_{A}(\theta, 2), D_{A}(\eta, 2)\right)} \leq C t^{-1+\theta-\eta}$ for $0<t \leq T$, see 
[10, p. 51]. Using the Hölder inequality we have for $0<t \leq T$

$$
\begin{aligned}
& \int_{0}^{t}\|(\beta I-A) S(t-s) f(s) \pi\|_{D_{A}(\eta, 2)} d s \\
& \leq C\|\pi\|_{D_{A}(\theta, 2)} \int_{0}^{t}(t-s)^{-1+\theta-\eta}|f(s)| d s \\
& \leq C\|\pi\|_{D_{A}(\theta, 2)}\left(\int_{0}^{t} s^{(-1+\theta-\eta) p^{\prime}} d s\right)^{1 / p^{\prime}}\left(\int_{0}^{t}|f(s)|^{p} d s\right)^{1 / p}<\infty .
\end{aligned}
$$

The next two lemmas concern the Laplace transform of the mild solution $\psi$ to (30).

Lemma 8.4. Let a satisfy Hypothesis 3.10 with $0<\eta<\theta<1$. Let $f$ belong to $L^{p}[0, T]$ for every $T>0$ where $1 \leq p<\infty$. Then the Laplace transform $\hat{\psi}:(\beta, \infty) \rightarrow D_{A}(\eta, 2)$ of the mild solution $\psi$ to (30) is well-defined and

$$
\hat{\psi}(s)=\hat{f}(s)\left(s I-A_{-1}\right)^{-1}\left(\beta I-A_{-1}\right) \pi, \quad s>\beta .
$$

Proof. To prove that $\hat{\psi}$ is well-defined on $(\beta, \infty)$ we show that $\int_{0}^{\infty} e^{-s t} \psi(t) d t$ exists as a Bochner integral in $D_{A}(\eta, 2)$ for $s>\beta$. Combining semigroup and interpolation theory it follows that there exists $C>0$ such that

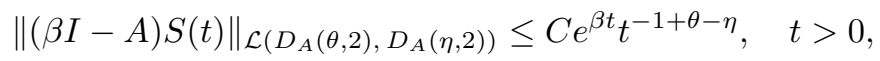

see $\left[\mathbf{1 0}\right.$, p. 51]. Using that the function $t \mapsto e^{\beta t} t^{\alpha}$ with $\alpha>-1$ has Laplace transform $s \mapsto\left(\Gamma(\alpha+1) /(s-\beta)^{\alpha+1}\right)$ on $(\beta, \infty)$ and that the Laplace transform of a convolution is the product of the individual Laplace transforms, we find that for $s>\beta$

$$
\begin{aligned}
\int_{0}^{\infty} & \left\|e^{-s t} \psi(t)\right\|_{D_{A}(\eta, 2)} d t \\
& \leq \int_{0}^{\infty} e^{-s t}\left(\int_{0}^{t}\|(\beta I-A) S(t-\sigma) f(\sigma) \pi\|_{D_{A}(\eta, 2)} d \sigma\right) d t \\
& \leq C\|\pi\|_{D_{A}(\theta, 2)} \int_{0}^{\infty} e^{-s t}\left(\int_{0}^{t} e^{\beta(t-\sigma)}(t-\sigma)^{-1+\theta-\eta}|f(\sigma)| d \sigma\right) d t \\
& =C\|\pi\|_{D_{A}(\theta, 2)} \frac{\Gamma(\theta-\eta)}{(s-\beta)^{\theta-\eta}} \widehat{|f|}(s)<\infty .
\end{aligned}
$$


By semigroup theory we have $\left(s I-A_{-1}\right)^{-1}=\int_{0}^{\infty} e^{-s t} S_{-1}(t) d t$ for $s>(\beta / 4)$, see $[\mathbf{1 0}$, p. 40]. Using Fubini's theorem by the above, we therefore have for $s>\beta$

$$
\begin{aligned}
\hat{\psi}(s) & =\int_{0}^{\infty} e^{-s t}\left(\int_{0}^{t}(\beta I-A) S(t-\sigma) f(\sigma) \pi d \sigma\right) d t \\
& =\int_{0}^{\infty} e^{-s \sigma} f(\sigma)\left(\int_{\sigma}^{\infty} e^{-s(t-\sigma)}(\beta I-A) S(t-\sigma) \pi d t\right) d \sigma \\
& =\left(\int_{0}^{\infty} e^{-s \sigma} f(\sigma) d \sigma\right)\left(\int_{0}^{\infty} e^{-s t}(\beta I-A) S(t) \pi d t\right) \\
& =\hat{f}(s) \int_{0}^{\infty} e^{-s t} S_{-1}(t)\left(\beta I-A_{-1}\right) \pi d t \\
& =\hat{f}(s)\left(s I-A_{-1}\right)^{-1}\left(\beta I-A_{-1}\right) \pi .
\end{aligned}
$$

Lemma 8.5. Let a satisfy Hypothesis 3.10 with $0<\eta<\theta<1$. Let $f$ belong to $L^{p}[0, T]$ for every $T>0$ where $1 \leq p<\infty$. Then $J(\hat{\psi}(s))=\frac{1}{s \hat{a}(s)} \hat{f}(s)$ for $s>\beta$.

Proof. Let $s>\beta$. Let $\pi_{n} \in D(A)$ be defined by $\pi_{n}:=n(n I-$ $A)^{-1} \pi$ for $n=1,2, \ldots$. It is a consequence of semigroup and interpolation theory that $\lim _{n \rightarrow \infty}\left\|\pi_{n}-\pi\right\|_{\left.D_{A} \theta, 2\right)}=0$, which implies $\lim _{n \rightarrow \infty}\left\|(\beta I-A) \pi_{n}-\left(\beta I-A_{-1}\right) \pi\right\|_{\left(H_{-1}, H\right)_{\theta, 2}}=0$ and hence,

$$
\lim _{n \rightarrow \infty}\left\|(s I-A)^{-1}(\beta I-A) \pi_{n}-\left(s I-A_{-1}\right)^{-1}\left(\beta I-A_{-1}\right) \pi\right\|_{D_{A}(\theta, 2)}=0 .
$$

As $D_{A}(\theta, 2)$ is continuously embedded in $D_{A}(\eta, 2)$, see $[\mathbf{1 0}, \mathrm{p} .16]$, this implies that

$$
\lim _{n \rightarrow \infty}\left\|(s I-A)^{-1}(\beta I-A) \pi_{n}-\left(s I-A_{-1}\right)^{-1}\left(\beta I-A_{-1}\right) \pi\right\|_{D_{A}(\eta, 2)}=0 .
$$

Since $J: D_{A}(\eta, 2) \rightarrow \mathbf{C}$ is continuous by Remark 3.12 , it follows that

$$
\lim _{n \rightarrow \infty} J\left((s I-A)^{-1}(\beta I-A) \pi_{n}\right)=J\left(\left(s I-A_{-1}\right)^{-1}\left(\beta I-A_{-1}\right) \pi\right) .
$$


To calculate the lefthand side of (31) we observe that Proposition 4.7 implies that $\pi_{n}(\kappa)=n \frac{\pi(\kappa)+p_{n}}{\kappa+n}$ for $\kappa \geq 0$ and $n=1,2, \ldots$ with

$$
p_{n}=\frac{1}{n \hat{a}(n)} \int_{[0, \infty)} \frac{\kappa \pi(\kappa)}{\kappa+n} \nu(d \kappa)=\frac{1}{n-\beta}\left(\frac{1}{\beta \hat{a}(\beta)}-\frac{1}{n \hat{a}(n)}\right) .
$$

By Proposition 4.7 and straightforward calculation it follows that

$$
\begin{aligned}
J\left((s I-A)^{-1}(\beta I-A) \pi_{n}\right) \\
=\frac{1}{s \hat{a}(s)} \int_{[0, \infty)} \frac{\kappa}{\kappa+s}(\beta I-A) \pi_{n}(\kappa) \nu(d \kappa) \\
=\frac{1}{s \hat{a}(s)} \int_{[0, \infty)} \frac{\kappa}{\kappa+s}\left(n \pi(\kappa)+(\beta-n) n \frac{\pi(\kappa)+p_{n}}{\kappa+n}\right) \nu(d \kappa) \\
=\frac{1}{s \hat{a}(s)} \frac{1}{\hat{a}(n)} \int_{[0, \infty)} \frac{\kappa}{\kappa+s} \frac{1}{\kappa+n} \nu(d \kappa) \\
=\frac{1}{s \hat{a}(s)} \frac{n}{n-s}-\frac{1}{\hat{a}(n)} \frac{1}{n-s} .
\end{aligned}
$$

It is a consequence of Lebesgue's dominated convergence theorem that $\lim _{n \rightarrow \infty} \hat{a}(n)=0$. Furthermore, the monotone convergence theorem combined with Lemma 4.1(iii) implies that $\lim _{n \rightarrow \infty} n \hat{a}(n)=+\infty$. Therefore $\lim _{n \rightarrow \infty}(n-s) \hat{a}(n)=+\infty$ and hence,

$$
\begin{aligned}
\lim _{n \rightarrow \infty} J\left((s I-A)^{-1}(\beta I-A) \pi_{n}\right) & =\lim _{n \rightarrow \infty} \frac{1}{s \hat{a}(s)} \frac{n}{n-s}-\frac{1}{\hat{a}(n)} \frac{1}{n-s} \\
& =\frac{1}{s \hat{a}(s)} .
\end{aligned}
$$

Combined with (31) this shows that $J\left(\left(s I-A_{-1}\right)^{-1}\left(\beta I-A_{-1}\right) \pi\right)=$ $\frac{1}{s \hat{a}(s)}$. Using Lemma 8.4 this proves that

$$
J(\hat{\psi}(s))=\hat{f}(s) J\left(\left(s I-A_{-1}\right)^{-1}\left(\beta I-A_{-1}\right) \pi\right)=\frac{1}{s \hat{a}(s)} \hat{f}(s) .
$$

Now we are in a position to prove Theorem 3.17. 
Proof. From Proposition 8.3 and the continuity of $J: D_{A}(\eta, 2) \rightarrow \mathbf{C}$ by Remark 3.12 it is clear that $u$ given by (12) satisfies the regularity properties stated in the theorem and hence, $u$ is Borel measurable. Moreover, $u$ satisfies Definition 3.2(ii) by the fact that $u(t)=0$ for $t \leq 0$, that $u$ belongs to $L^{1}[0, T]$ for every $T>0$, and that $a$ is continuous, see [9, p. 38]. To show that $u$ satisfies Definition 3.2(iii) and (iv) we calculate the function $v$. Since $J: D_{A}(\eta, 2) \rightarrow \mathbf{C}$ is continuous and $\int_{0}^{\infty} e^{-s t} \psi(t) d t$ exists for $s>\beta$ in $D_{A}(\eta, 2)$ by Lemma 8.4, we have for $s>\beta$ using Lemma 8.5

$$
\hat{u}(s)=\int_{0}^{\infty} e^{-s t} J(\psi(t)) d t=J(\hat{\psi}(s))=\frac{1}{s \hat{a}(s)} \hat{f}(s),
$$

that is, $\hat{a}(s) \hat{u}(s)=(1 / s) \hat{f}(s)$. As the function $t \mapsto \int_{0}^{t} f(s) d s$ has Laplace transform $s \mapsto 1 / s \hat{f}(s)$ and the Laplace transform of a convolution is the product of the individual Laplace transforms, the inversion theorem for Laplace transforms implies that $v(t)=\int_{0}^{t} a(t-s) u(s) d s=$ $\int_{0}^{t} f(s) d s$ for $t>0$. It follows that $u$ satisfies Definition 3.2(iii) and (iv).

Acknowledgments. The authors thank the referees for valuable comments. Wolfgang Desch would like to thank the Delft University of Technology for the kind hospitality. Philippe Clément and Krista Homan would like to thank the Karl-Franzens-Universität Graz for the kind hospitality.

\section{ApPendix}

A. For $f, g:(0, \infty) \rightarrow \mathbf{R}$ we let $f * g:(0, \infty) \rightarrow \mathbf{R}$ denote the function $(f * g)(t):=\int_{0}^{t} f(t-s) g(s) d s$ for $t>0$. Recall that the function $g_{\rho}:(0, \infty) \rightarrow \mathbf{R}$ with $\rho>0$ is given by (13).

For a proof of each of the following two propositions we refer to $[\mathbf{3}]$.

Proposition A.1. Let $0<\rho<1$ and $1 \leq p<\infty$. Let $f$ belong to $L^{p}[0, T]$ for some $T>0$. Then the following holds:

(i) If $p=1$, then $g_{\rho} * f$ belongs to $L^{q}[0, T]$ for every $1 \leq q<\frac{1}{1-\rho}$ 
and there exists $c>0$ depending on $\rho$ and $q$ such that

$$
\left\|g_{\rho} * f\right\|_{L^{q}[0, T]} \leq c\|f\|_{L^{p}[0, T]}
$$

(ii) If $1<p<\frac{1}{\rho}$, then $g_{\rho} *$ fbelongs to $L^{q}[0, T]$ for every $1 \leq q \leq$ $\frac{p}{1-\rho p}$ and there exists $c>0$ depending on $\rho, p$, and $q$ such that (32) holds;

(iii) If $p=\frac{1}{\rho}$, then $g_{\rho} * f$ belongs to $L^{q}[0, T]$ for every $1 \leq q<\infty$ and there exists $c>0$ depending on $\rho$ and $q$ such that (32) holds;

(iv) If $\frac{1}{\rho}<p<\infty$, then $g_{\rho} * f$ belongs to $C_{0}^{\rho-\frac{1}{p}}[0, T]$ and there exists $c>0$ depending on $\rho$ and $p$ such that

$$
\left\|g_{\rho} * f\right\|_{L^{\infty}[0, T]}+T^{\rho-\frac{1}{p}}\left[g_{\rho} * f\right]_{\rho-\frac{1}{p}} \leq c T^{\rho-\frac{1}{p}}\|f\|_{L^{p}[0, T]},
$$

where

$$
\left[g_{\rho} * f\right]_{\rho-\frac{1}{p}}:=\sup \left\{\frac{\left|\left(g_{\rho} * f\right)(t)-\left(g_{\rho} * f\right)(s)\right|}{(t-s)^{\rho-\frac{1}{p}}} ; \quad 0 \leq s<t \leq T\right\} .
$$

Proposition A.2. Let $(X,\langle\cdot, \cdot\rangle)$ be a real or complex Banach space. Let $A: D(A) \subseteq X \rightarrow X$ be a linear operator that is the infinitesimal generator of an analytic semigroup $\{S(t)\}_{t \geq 0}$. Let $1 \leq p<\infty$, $0<\alpha<1,1 \leq r \leq \infty$, and let $f$ belong to $L^{p}\left([0, T] ; D_{A}(\alpha, r)\right)$ for every $T>0$. Then the following holds:

(i) If $x$ belongs to $D_{A}(\alpha, r)$, then $t \mapsto S(t) x$ belongs to $C^{\alpha}([0, T] ; X)$ for every $T>0$;

(ii) If $x$ belongs to $D(A)$, then $t \mapsto S(t) x$ belongs to $C^{1-\alpha}([0, T]$; $\left.D_{A}(\alpha, r)\right)$ for every $T>0$;

(iii) Let $0<\gamma \leq \alpha$. If $x$ belongs to $D_{A}(\alpha, r)$, then $t \mapsto S(t) x$ belongs to $C^{\alpha-\gamma}\left([0, T] ; D_{A}(\gamma, r)\right)$ for every $T>0$; 
(iv) Let $0<\gamma<\alpha$. If $p=1$, then $t \mapsto \int_{0}^{t} A S(t-s) f(s) d s$ belongs to $L^{q}\left([0, T] ; D_{A}(\gamma, r)\right)$ for every $1 \leq q<\frac{1}{1-(\alpha-\gamma)}$ and $T>0$

(v) Let $0<\gamma<\alpha$. If $1<p<\frac{1}{\alpha-\gamma}$, then $t \mapsto \int_{0}^{t} A S(t-s) f(s) d s$ belongs to $L^{q}\left([0, T] ; D_{A}(\gamma, r)\right)$ for every $1 \leq q \leq \frac{p}{1-(\alpha-\gamma) p}$ and $T>0$

(vi) Let $0<\gamma<\alpha$. If $p=\frac{1}{\alpha-\gamma}$, then $t \mapsto \int_{0}^{t} A S(t-s) f(s) d s$ belongs to $L^{q}\left([0, T] ; D_{A}(\gamma, r)\right)$ for every $1 \leq q<\infty$ and $T>0$;

(vii) Let $0<\gamma<\alpha$. If $\frac{1}{\alpha-\gamma}<p<\infty$, then $t \mapsto \int_{0}^{t} A S(t-s) f(s) d s$ belongs to $C_{0}^{\alpha-\gamma-\frac{1}{p}}\left([0, T] ; D_{A}(\gamma, r)\right)$ for every $T>0$.

B. For a proof of the next proposition we refer to $[\mathbf{3}]$.

Proposition B.1. Let $u_{0}: \mathbf{R} \rightarrow \mathbf{R}$ be given by $u_{0}(t):=\gamma e^{\beta t}$ for $t \in \mathbf{R}$ where $\gamma \in \mathbf{R}$. Then problem (1) with $f$ identically zero admits a unique solution and

$$
u(t)= \begin{cases}u_{0}(t)-\beta \hat{a}(\beta) \int_{0}^{t} b(t-s) u_{0}(s) d s & t>0, \\ u_{0}(t) & t \leq 0\end{cases}
$$

where $b:(0, \infty) \rightarrow \mathbf{R}$ is the integral resolvent of the first kind of $a$, see $(2)$.

\section{REFERENCES}

1. H. Amann, Linear and quasilinear parabolic problems, Vol. I: Abstract linear theory, Birkhäuser Verlag, Basel, 1995.

2. W. Arendt, A. Grabosch, G. Greiner, U. Groh, H.P. Lotz, U. Moustakas, R. Nagel, F. Neubrander and U. Schlotterbeck, One-parameter semi-groups of positive operators, Springer-Verlag, Berlin, 1986.

3. Ph. Clément, W. Desch and K.W. Homan, An analytic semigroup setting for a class of Volterra equations, in Optimierung und Kontrolle, Karl-FranzensUniversität und Technische Universität Graz, SFB F003 Bericht Nr. 242, 2002. 
4. Ph. Clément, H.J.A.M. Heijmans, S. Angenent, C.J. van Duijn and B. de Pagter, One-parameter semigroups, North-Holland Publ. Co., Amsterdam, 1987.

5. G. Da Prato and J. Zabczyk, Stochastic equations in infinite dimensions, Cambridge Univ. Press, Cambridge, 1992.

6. W. Desch and R. Grimmer, Singular relaxation moduli and smoothing in threedimensional viscoelasticity, Trans. Amer. Math. Soc. 314 (1989), 381-404.

7. W. Desch and K.W. Homan, Pointwise versions of solutions to Cauchy problems in $L^{p}$-spaces in Optimierung und Kontrolle, Karl-Franzens-Universität und Technische Universität Graz, SFB F003 Bericht Nr. 250, 2002.

8. W. Desch and R.K. Miller, Exponential stabilization of Volterra integral equations with singular kernels, J. Integral Equations Appl. 1 (1988), 397-433.

9. G. Gripenberg, S-O. Londen and O.J. Staffans, Volterra integral and functional equations, Cambridge Univ. Press, Cambridge, 1990.

10. A. Lunardi, Analytic semigroups and optimal regularity in parabolic problems, Birkhäuser Verlag, Basel, 1995.

11. R.K. Miller, Linear Volterra integrodiffrential equations as semigroups, Funk. Ekvac. 17 (1974), 39-55.

12. A. Pazy, Semigroups of linear operators and applications to partial differential equations, Springer-Verlag, New York, 2nd ed., 1983.

13. J. Prüss, Evolutionary integral equations and applications, Birkhäuser Verlag, Basel, 1993.

14. O.J. Staffans, Well-posedness and stabilizability of a viscoelastic equation in energy space, Trans. Amer. Math. Soc. 345 (1994), 527-575.

15. E.M. Stein, Topics in harmonic analysis related to the Littlewood-Paley theory, Princeton Univ. Press, Princeton, NJ, 1970.

16. $\mathrm{H}$. Triebel, Interpolation theory, function spaces, differential operators, North-Holland Publ. Co., Amsterdam, 1978.

17. D.V. Widder, The Laplace transform, Princeton Univ. Press, Princeton, NJ, 1941.

18. K. Yosida, Functional analysis, Springer-Verlag, Berlin, 1995.

E-mail address: clement@its.tudelft.nl

E-mail address: georg.desch@uni-graz.at

Department of Applied Mathematical Analysis, Delft University of Technology, P.O. Box 5031, 2600 GA Delft, The Netherlands

E-mail address: k.w.homan@its.tudelft.nl 